\title{
An additively manufactured silicone polymer : Thermo-viscoelastic experimental study and computational modelling
}

\author{
Mokarram Hossain ${ }^{\mathrm{a}, *}$, Zisheng Liao ${ }^{\mathrm{a}, \mathrm{b}}$ \\ ${ }^{a}$ Zienkiewicz Centre for Computational Engineering, College of Engineering, Swansea University, SA1 8EN, United Kingdom \\ ${ }^{b}$ School of Civil Engineering and Transportation, South China University of Technology, 510640 Guangzhou, Guangdong, China
}

\begin{abstract}
The additive manufacturing (AM) is a new paradigm across various disciplines of engineering sciences. Despite significant advances in the areas of hard material printings, the options for 3D printed soft materials are still limited. Most of the existing 3D printed polymers are in the areas of acrylics and polyurethanes or their composites. Recently emerged Digital Light Synthesis (DLS) technology hugely accelerates the additive manufacturing of soft polymers. A DLS-inspired 3D printer uses a continuous building technique instead of a layer-by-layer approach, where the curing process is activated by an ultra-violet (UV) light. In this contribution, a DLS-based digitally printed silicone (SIL30) is experimentally characterized. To understand polymer's temperature-dependent mechanical responses, an extensive thermo-viscoelastic experimental characterisation at various strain rates under tensile deformation and temperature fields from $-20^{\circ} \mathrm{C}$ to $60^{\circ} \mathrm{C}$ is performed. The study reveals significant effects of time-and temperature-dependency on the mechanical responses of the 3D printed silicone. Motivated by the thermo-mechanical results of the polymer, a thermodynamically consistent constitutive model at large strain is devised. Afterwards, the model is calibrated to the data that results in the identification of relevant parameters. The model predicts the experimental results with a good accuracy. 3D printed soft polymers are major candidates in designing complex and intricate architectured metamaterials for biomedical applications. Hence, a comprehensive thermo-mechanical experimental study and subsequent constitutive modelling will facilitate in designing and simulating more complex cellular metamaterials using 3D printed silicones.
\end{abstract}

Keywords: 3D printing, Silicone polymer (SIL30), Digital Light Synthesis (DLS), Additive manufacturing, Thermo-viscoelastic modelling, Metamaterials

\section{Introduction}

Among other soft materials, silicone polymers have many outstanding properties. These include, but not limited to, biocompatibility (ideal for medical applications), good transparency, chemical stability and resistance to natural ageing, thermal stability over a wide range of temperature profiles, ability to withstand fire without releasing toxic and irritating fumes, electrical insulation, and good elasticity. Silicones are less susceptible to react chemically with other materials. Hence, they are more preferred polymers in manufacturing bio-medical accessories and devices, e.g., for hearing aids, tubing, peristaltic pumps, breast prosthesis for breast cancer patients, catheters and cardiovascular devices [6,26,27]. Thanks to their matchable stiffnesses

*mokarram.hossain@swansea.ac.uk

Email addresses: mokarram.hossain@swansea.ac.uk (Mokarram Hossain), 1.zisheng@mail.scut.edu.cn (Zisheng Liao)

Accepted for publication in Additive Manufacturing 
with biological tissues, silicones and their composites are major materials as substrates in mechanobiology, see $[55,56]$. More recently, silicones and their blends and composites are becoming major ingredients in several emerging sectors such as soft robotics, soft and flexible electronics $[10,11,30]$. In addition to silicones' outstanding mechanical properties, reduced dissipative behaviour, large stretchability, temperature insensitivity, pure silicones or their composites and blends become ideal materials in flexible-sensors, soft robotics, and energy harvesters $[2,15,17,32,42,60]$.

Owing to a wide range of applications across many disciplines, experimental study and constitutive modelling of silicone polymers, particularly at finite strain, attract considerable attention. In an effort to understand the mechanical behaviour of two commercially available silicones (B452 and Sil8800), Shergold et al. [53] performed an experimental study under compression tests at various strain rates spanning from $10^{-3}$ to $10^{3} / \mathrm{s}$. Their aim was to compare mechanical responses of human skins with the two aforementioned soft silicones. The study concluded that a strain rate-dependency of silicones could only be observed when the strain rate exceeds 40 /s. To understand silicones' thermo-mechanical behaviour, in contrast to isothermal experimentations of Shergold et al. [53], Rey et al. [47] conducted an extensive investigation on the influence of temperature on elasticity, Mullins stress-softening, loading-unloading cyclic behaviour, and stress exhibited entropic elastic behaviour above $150^{\circ} \mathrm{C}$. Guo et al. [20] conducted high strain rate tests on filled silicones using a split Hopkinson tension bar (SHTB) with strain rates ranging from $10^{-3}$ to $1400 / \mathrm{s}$. They further conducted high strain-rate tensile tests at various temperatures which reveal that the materials stiffnesses increase with increased strain rates and decrease with increasing temperature profiles. Bernardi et al. [6] conducted a couple of experiments including uniaxial and biaxial tensile deformation states for several silicone polymers. Furthermore, they developed constitutive models at large strains to predict stress-strain responses of silicones. Ecoflex, a commercially available silicone, becomes an ideal choice in bio-medical engineering and stretch-based sensors applications thanks to its high stretchability with a minimum viscosity [2]. In an effort to quantify Ecoflex thermo-viscoelastic characteristics, very recently, Liao et al. [35, 36] conducted an extensive study on the material of various Shore hardnesses. The Platinum-catalised, room temperature-cured polymer shows very insignificant strain rate-and temperature-sensitive responses. Based on the experiment study, they further developed a Shore-dependent constitutive framework coupled with the pseudo-elastic softening behaviour significantly observed in Ecoflex [35].

The additive manufacturing (AM) or 3D printing is relatively a new field of digital manufacturing. It not only revolutionises the area of rapid prototyping but also facilitates the production of multi-characteristics, multi-purpose, complex metamaterials. The 3D printing closes the gaps between ideas and realities by making prototypes within a very short span of time. Using a wide range of AM techniques, three-dimensional structures of complex and intricate geometries with a very high precision and surface finish can be produced which are simply impossible with traditional manufacturing processes. Therefore, the AM has been drawing unprecedented and ever-increasing interests from industry and academia alike. New materials and devices made of additive manufacturing techniques have fast increasing applications in almost all areas, e.g., construction, apparels, dentistry, medicine, flexible electronics, automotive, robotics, military, oceanography, aerospace, to mention a few $[38,54,55,56]$. A recent report revealed that almost half of the additively manufactured materials are soft polymers that can be printed with a myriad of properties [61]. Several 3D printing companies such as Stratasys, Carbon3D, ACEO3D, RepRap, Protolabs, Formlabs, Materialise, SpectroPlast produce a wide range of soft polymeric materials that can sustain extremely large deformations before failure. Note that in almost all of the 3D printed soft polymers, an UV light is used for the 
hardening (curing) process. Soft photo-curing resins (uncured liquid monomers) could be acrylics, silicone, and polyurethane resins, whereby several AM techniques such as Inkjet printing, Drop-on Demand (DOD), Stereolithography Apparatus (SLA), Digital Light Processing (DLP), and Digital Light Synthesis (DLS) (previously known as Continuous Liquid Interface Production or CLIP) have widely been used. Among other processes for soft polymers and their composites, the recently invented DLS helps reducing the design and fabrication processes from several hours to a few minutes or even less [38, 55, 62].

Similar to experimental characterizations and numerical modelling of additively manufactured hard materials such as metal and alloys [13], recently emerged digitally printed soft polymers also receive significant attention thanks to the latter myriad of applications [19, 38, 39, 64]. However, thermo-mechanical experimental studies of 3D printed soft and flexible materials at large deformations are rare in the literature. Very recently, few experimental works only deal with small strain-based characterizations such as the determination of shear modulus, bulk modulus, loss modulus etc [7, 48]. For instance, Reichl and Inman [48] characterized viscoelastic properties of 3D printed soft polymers such as frequency and temperaturedependent complex moduli. Note that such experimental techniques based on linearized strain theories provide inadequate data for materials undergoing large strains. 3D printed soft polymers are promising inaterials in soft robotics and stretch-based sensor applications $[2,10,11]$. To develop reliable and aceurate mathematical models that can predict the responses of soft materials over different time scales and temperatures, experimental characterizations of time- and temperature-dependent material behaviour especially dissipative and relaxation behaviour are very much essential [14]. To fulfil the gaps in experimental characterizations of digitally manufactured polymers (e.g.,Tangoflex series), Slesarenko and Rudykh [54], Liu and Li [37] performed strain rate-dependent experiments on Tangoflex-Verowhite at large strains. Very recently, Abayazid and Ghajari [4] characterised the mechanical responses at large strains for two PolyJet elastomers, namely Agilus30 (A30) and Tango+ (T+). Hossain et al. [25] conducted an extensive study of a 3D printed soft elastomeric polyurethane (EPU) manufactured by the DLS technique. Moreover, they developed a viscoelastic model incorporating nonlinear dependencies of viscosities significantly observed in of FDM (fused deposition modelling)-based thermoplastic polymers and their composites have actively been investigated in the literature [1, 17, 43, 49, 57]. However, chemical compositions and printing processes for the thermoplastics are completely different from the 3D printed soft and flexible elastomeric polymers.

Thermo-viscoelastic experimental characterizations at large strains of any 3D printed silicone polymer are rare in the literature. The current contribution addresses two major aspects of a commercially available 3D printed silicone, SIL30. Firstly, a comprehensive thermo-viscoelastic study of the polymer is demonstrated. For that, some classical experiments which are paramount in understanding a viscoelastic polymer at large strains are chosen. These include Mullins effect tests, cyclic tests, quasi-static tests, and two types of relaxation tests under thermo-mechanical loads. Secondly, based on the experimental study presented in the first half, a thermodynamically consistent geometrically nonlinear thermo-mechanical constitutive model is proposed. Note that experimental results presented herein are obtained from homogeneous tensile deformations. Therefore, the thermo-viscoelastic model formulated in a three-dimensional setting needs to be devised as a set of one-dimensional equations. Afterwards, with the help of the data, model parameters are identified by an optimization technique. For the model validation, other sets of experimental data are used which are excluded in the parameter identification. It is worth to mention that some classical silicone polymers such as Ecoflex, Sylgard 184, RTV 141, RTV 3428, B452, and Sil8800 are temperature-cured polymers. They are cured either at room temperature or at elevated temperatures. Hence, they are not suit- 


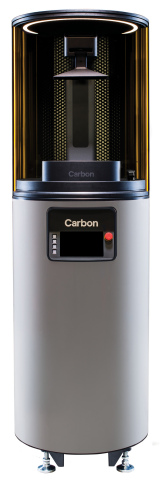

(a)

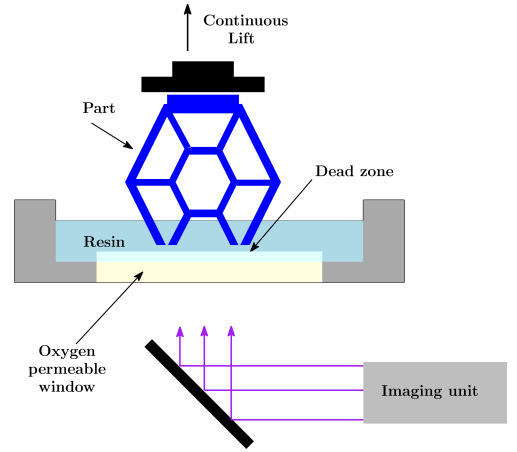

(b)

Figure 1. Carbon3D DLS technology : (a) Carbon3D's latest 3D printer CarbonM2, (b) a sketch describing the basic functionality of the DLS technology

able for vat-based 3D printing in which an ultra-violet (UV) light is used as the curing initiator. UV-cured silicones are essential for the manufacturing of bespoke and intricate geometries especially in biomedical engineering in which many traditional silicones are not suitable. The current silicone will fill up the gaps.

The manuscript has five sections. We briefly describe the experimental set-up and procedures which are followed to conduct relevant tests in Section 2. In the same section, we present and analyse all experimental results. A novel thermo-viscoelastic computational model is proposed for the additively manufactured polymer in Section 3. Therein, for material parameters identification, all equations are decomposed in one-dimensional forms. In Section 4, validations of the model are demonstrated and discussed. Section 5 summarises all major findings of the current work and gives an outlook for future works.

\section{Thermo-viscoelastic experiments and results}

Here, a comprehensive thermo-mechanical experimental study for a 3D printed silicone (SIL30) is performed using an Instron machine. In the case of thermo-viscoelastic experiments, the main frame of the machine is inserted in the chamber, cf. Fig. 2(a). According to some classical literature on experimental study and constitutive modelling for thermo-viscoelastic polymeric materials, e.g., Behnke et al. [5], Lion [33], Dippel et al. [16], Hossain et al. [23], Liao et al. [34, 35], Amin et al. [3], a couple of distinct experiments are required which will be discussed in the following sections.

\subsection{Working principle for the DLS process}

The silicone polymer investigated in this study is manufactured by the recently invented Digital Light Synthesis (DLS) technique. Hence, a very short note on the DLS process is given here. Earlier, the 3D printing for soft polymers and their composites mainly uses layer-by-layer or dot-by-dot building processes such as Inkjet printing, Stereolithography Apparatus (SLA), and Digital Light Projection (DLP). These techniques are time-consuming AM processes. The DLS (previously known as Continuous Liquid Interface Production or CLIP) technology invented by DeSimone and co-workers [62] changes the arena of the traditional layer-by-layer or dot-by-dot building processes, particularly for soft elastomers. A DLS printer such as 


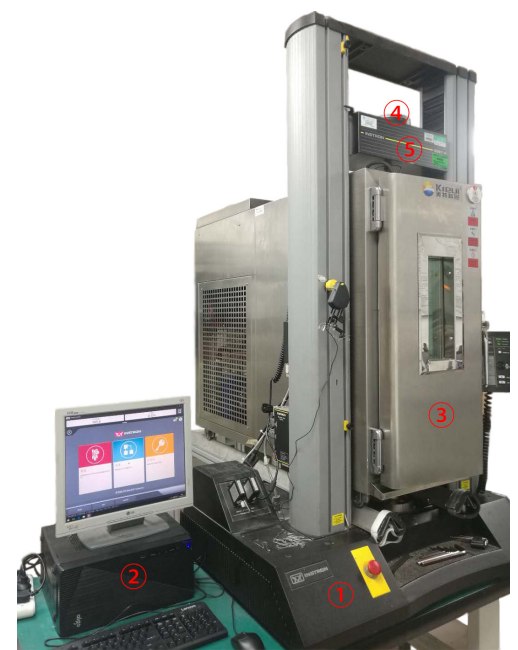

(a)

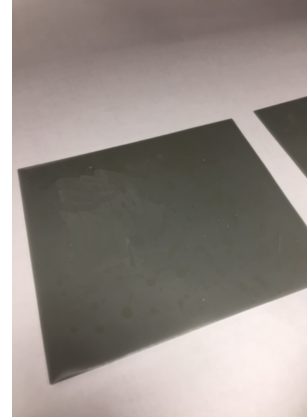

(b)

Figure 2. A set-up for the thermo-viscoelastic testing system with a 3D printed sample: (a) represents various sections of the system where, (1) indicates the key structure, (2) shows a computer that controls the machine. Moreover, (3) illustrates a closed chamber and (4) contains a force sensor, while (5) indicates the crosshead, (b) a bigger sample of $1 \mathrm{~mm}$ uniform thickness with a rectangular area of $100 \mathrm{~mm} \times 120 \mathrm{~mm}$.

Carbon3D M2 (Fig. 1(a)) starts the curing process using a bottom-up approach. There exists an oxygen inhibited dead-zone that prevents any adherence of the 3D part to the uncured part, see Fig. 1(b). The thin oxygen-permeable window is made of an amorphous Teflon film which has a high chemical resistance and gas transport potential. It has a very low refractive index with an excellent optical clarity. Such a thin film has an optical property that extends to the range of UV. When oxygen permeates through the window creating a very thin layer between the window and the printing part, known as the dead-zone, of uncured resin. The extremely thin layer of one-third of a human hair is the area responsible for Carbon3D's remarkable performance with isotropic 3D printed parts. In the dead-zone, oxygen prohibits lightening curing the resins situated closest to the window, thereby allowing for the continuous flow of liquid resins beneath the part [55].

\subsection{Sample preparation}

As mentioned earlier, in this study, we selected a DLS-based 3D printed silicone (SIL30) produced by Carbon3D ${ }^{\mathrm{TM} 1}$. Necessary samples of the silicone printed by a Carbon3D M2 were purchased from Paragon Rapid Technologies (UK), a local Production Network of the Carbon3D. In preparing specimens for uniaxial homogeneous tests, two techniques have been widely used in the area of soft polymers experimentation. First option is the so-called dog-bone samples that significantly reduce the grippers' (edge) effects due to the presence of enhanced areas at sample ends $[17,54,58]$. Second alternative route is the rectangular sample, in which a length to width ratio $\approx 10: 1$ is maintained throughout the sample length $[34,35,36,28,51,59]$. Due to excessive costs for the DLS 3D printed dog-bone samples, the second option for the specimen preparation is adopted in the current study. For that, a CAD (Computer Aided Design) geometry of $1 \mathrm{~mm}$ uniform thickness with a rectangular area of $100 \mathrm{~mm} \times 120 \mathrm{~mm}$ (Fig. 2(b)) is created. Afterwards, slices of $5 \mathrm{~mm}$

\footnotetext{
${ }^{1}$ https: / / www. carbon3d.com
} 


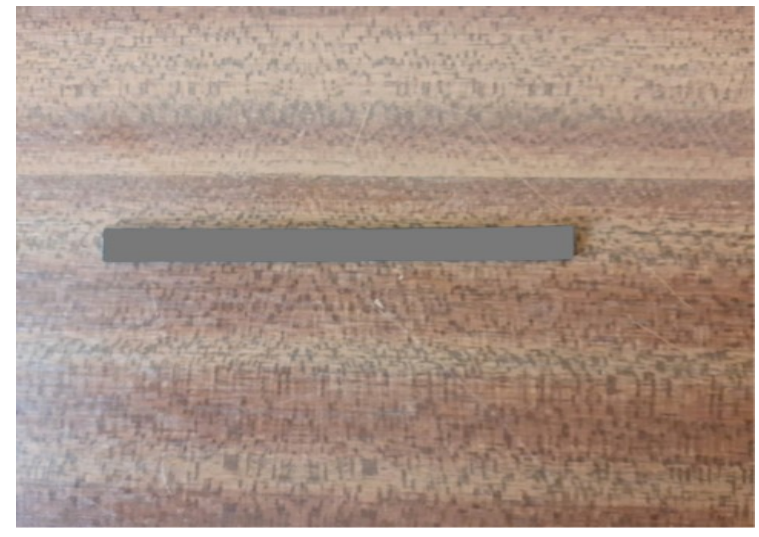

(a)

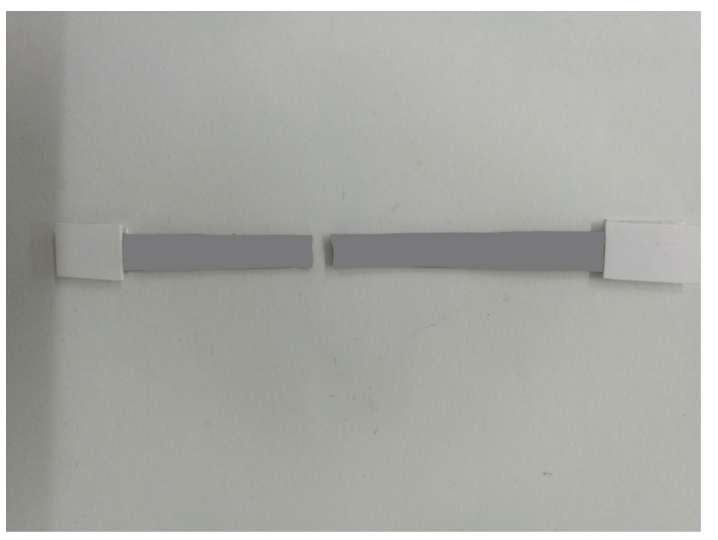

(b)

Figure 3. Rectangular sample preparation : (a) A $5 \mathrm{~mm}$ x $60 \mathrm{~mm}$ x $1 \mathrm{~mm}$ rectangular virgin sample (undeformed) before any test, (b) A sample with its place of failure (middle) after a uniaxial tensile test (deformed)

wide and $120 \mathrm{~mm}$ long are cut from the $100 \mathrm{~mm}$ x $120 \mathrm{~mm}$ big sample. Again each slice is divided into two pieces of $5 \mathrm{~mm}$ wide and $60 \mathrm{~mm}$ long. Finally, a rectangular sample becomes $5 \mathrm{~mm}$ x $60 \mathrm{~mm}$ x $1 \mathrm{~mm}$ (width $\mathrm{x}$ length $\mathrm{x}$ thickness) which will be around $40 \mathrm{~mm}$ long and $5 \mathrm{~mm}$ wide excluding the portions captured in the grippers. Such a dimension maintains 8:1 ratio (length: width). A similar sample dimension was taken previously in the case of soft polymers like SIL30 for testing a homogeneous tensile mode of deformation, see Liao et al. [34, 35, 36], Johlitz et al. [28], Sahu and Patra [51], Wissler and Mazza [59]. In order to prepare rectangular samples of $5 \mathrm{~mm} \times 60 \mathrm{~mm} \times 1 \mathrm{~mm}$, at first, desired lines are inscribed on the top of the big sample (100 mm x $120 \mathrm{~mm}$ ) using a soft ballpoint pen. Afterwards, a sharp trimmer (e.g., Dahle brand) is used to cut a desired sample. As shown in (Fig. 3(a)), samples are precisely cut. Moreover, sharp and long scissors are also used to cut the samples that result in less precise specimens, see (Fig. 3(b)). Regarding the sample failures, most of them fail at the middle, see (Fig. 3(b)). However, few samples fail at the gripper ends. Hence, in order to reduce gripper's influence, samples are glued with white papers at the ends, see (Fig. 3(b)). For large stretched tests, a specimen could possibly slide out from the grippers. Therefore, the layers of white papers used between the grippers and the specimen prevent sliding of specimens out of the grippers. Slesarenko and Rudykh [54] mentioned that 3D printed UV-cured soft polymers might have some time-dependent ageing phenomena that could affect their mechanical properties. Hence, in order to avoid any ageing, all tests are completed within the first month of the printed polymer.

\subsection{Experimental set-ups}

In order to perform thermo-mechanical tests, we use a purposively built temperature chamber, see Fig 2. The chamber can be regulated ranging from temperature $-50^{\circ} \mathrm{C}$ to $150^{\circ} \mathrm{C}$. The machine frame can move up to $800 \mathrm{~mm}$. However, the frame displacement is restricted to $200 \mathrm{~mm}$ when it is caged in the chamber. The machine has a crosshead speed of $8 \mathrm{~mm} / \mathrm{s}$. Bluehill ${ }^{\circledR}$, an Instron built-in software, is used to run the machine. For presenting experimental results in stress-strain formats, we use nominal stresses (also known as the first Piola-Kirchhoff stresses) and nominal strains. Subsequently, the time derivatives of the nominal strains will yield strain rates. The Instron machine has a maximum capacity of $\pm 50 \mathrm{~N}$ with a precision of $0.001 \mathrm{~N}$. A good reproducibility of experimental results is an important issue. Hence, we repeat each set of experiment at least three to five times and the average one is chosen. For detailed set-ups of the machine 


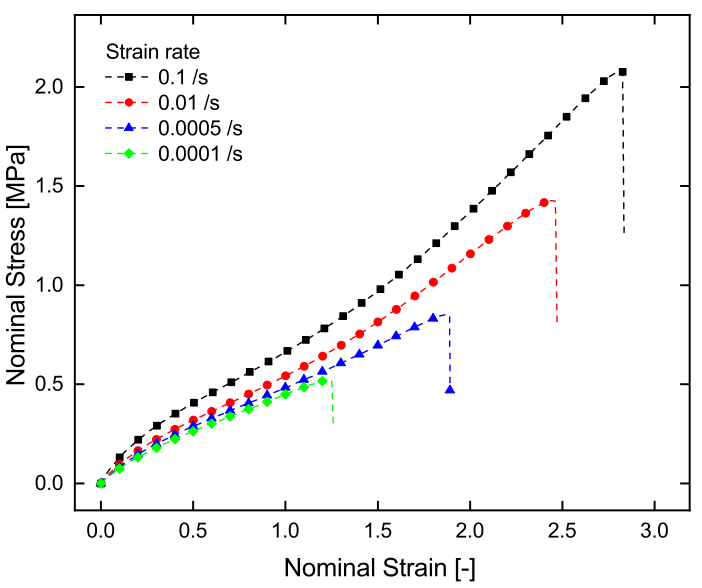

(a)

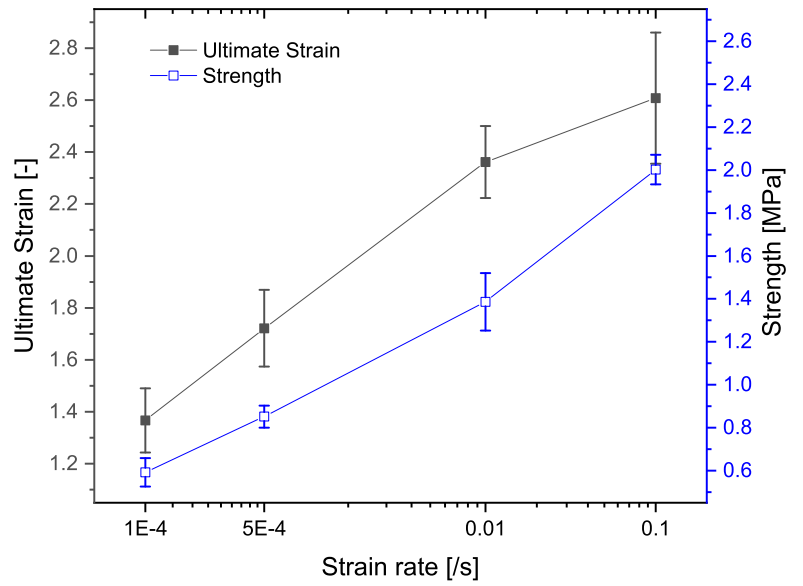

(b)

Figure 4. In search of stretchability of SIL30 silicone: (a) Elongation at break is highly dependent on strain rates, (b) stretchability and ultimate strength of SIL30 increase with the increase of strain rates (error bars are added to show the variations in experiments)

to conduct thermo-viscoelastic experiments, readers are referred to our previous works, e.g., Liao et al. $[34,36]$.

\subsection{Stretchability and Mullins effect of the $3 D$ printed silicone}

At first, our aim is to find the maximum elongation at break (stretchability) of SIL30. The polymer is expected to be a viscoelastic material, i.e., it will show a strain rate-dependent behaviour. Therefore, instead of a fixed machine speed, four strain rates, e.g., $0.0005 / \mathrm{s}, 0.01 / \mathrm{s}, 0.05 / \mathrm{s}$, and $0.1 / \mathrm{s}$ are selected to assess the stretchability of the material. Fig. 4 depicts that the material breaks at a lower strain with a slowest strain rate. This behaviour is very similar to some other 3D printed soft polymers. For example, PolyJet printed Tangoflex-Verowhite soft polymers become less stretchable with a slower strain rate, see [4, 37, 54]. From Fig. 4, it can be concluded that SIL30's elongation at breaks is around $250 \% \pm 20 \%$ (error bars are added to demonstrate the variations in experiments) strain with a strain rate of $0.01 / \mathrm{s}$. Moreover, the stress-strain curves demonstrate a significant nonlinearity within the strains, which is very similar to the classical S-shaped curve (hardening-softening-hardening) demonstrated by major elastomers, see [63]. Note that according to a manual supplied by Carbon3D [12], at 50\% and $100 \%$ strains, the polymer should yield 0.42 MPa and 0.69 MPa tensile stresses, respectively. A similar trend is observed in our experimental study where, $50 \%$ and $100 \%$ strains yield $0.41 \mathrm{MPa}$ and $0.70 \mathrm{MPa}$ tensile stresses (at a strain rate of 0.05 /s), respectively, see Fig. 6 . Hence, it can be concluded that the printing conditions of the samples we used in this study resemble the standard conditions of a Carbon3D M2 printer.

In order to verify whether a soft polymer shows any stress-softening or Mullins effect, a combination of two distinct loading-unloading cyclic experiments is required as frequently suggested in the literature $[18,58]$. The first category is the loading-unloading cyclic test which is conducted at a specified strain and strain rate. For this, a sample is stretched up to a certain point and is reloaded to the zero strain without any stopover between a loading and an unloading cycles. The same sample is reloaded from the zero strain without any delay between two consecutive full cycles. Most of the softening, which is characterized by a lower resulting stress for the same applied strain, appears after the first cycle. After a few cycles (values up to 10 are reported in the literature depending on the material nature), the material responses coincide during the 


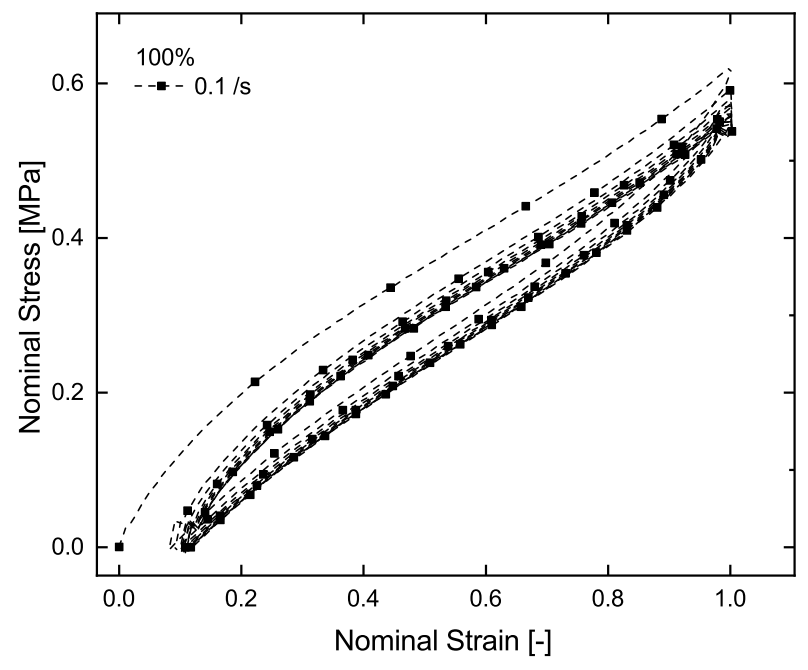

(a)

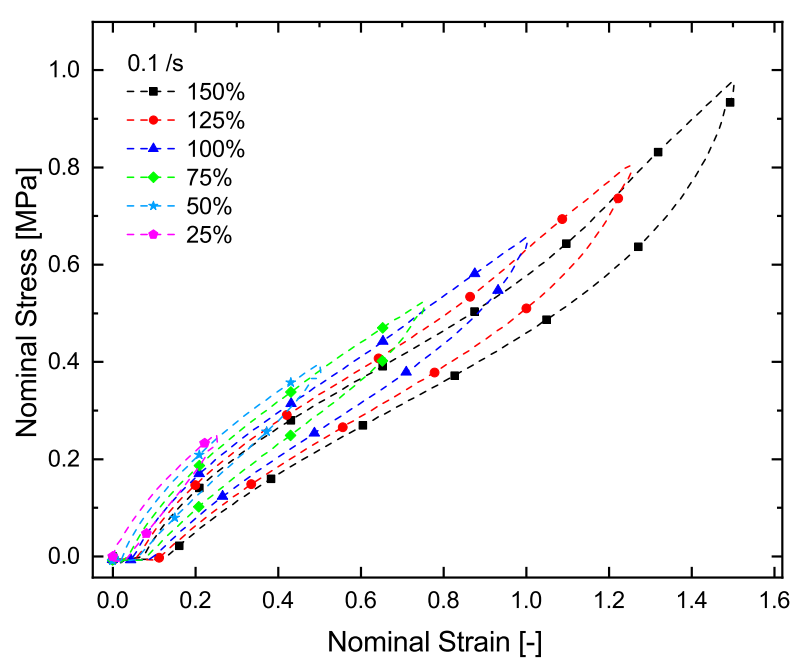

(b)

Figure 5. Mullins stress-softening tests: (a) Stress-softening test with several loading-unloading cycles at a fixed strain and strain rate, (b) repeated cycles at an increasing strain (@ 25\%) with a fixed strain rate on a virgin sample; Different colors indicate increasing loading-unloading paths

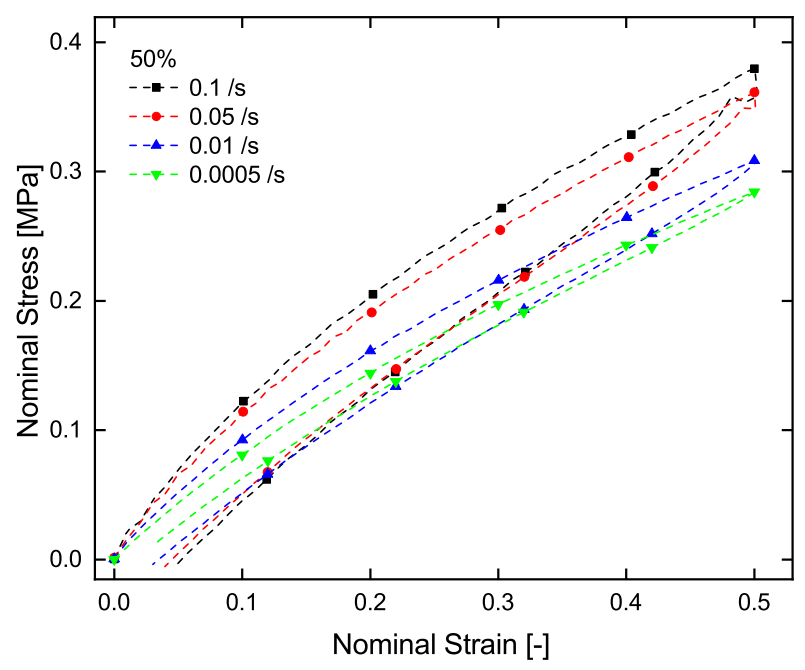

(a)

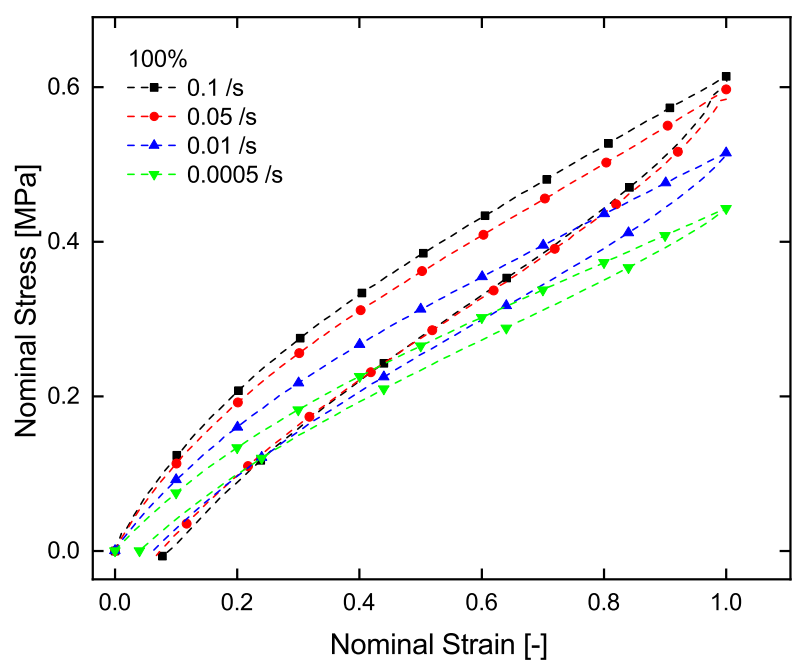

(b)

Figure 6. Loading-unloading cyclic experiments at four strain rates 0.1 /s, 0.05 /s, 0.01 /s, and 0.0005/s: (a) 50\% strain, (b) $100 \%$ strain. Within these strains, an initial strain hardening is observed followed by a strain softening up to $100 \%$ 


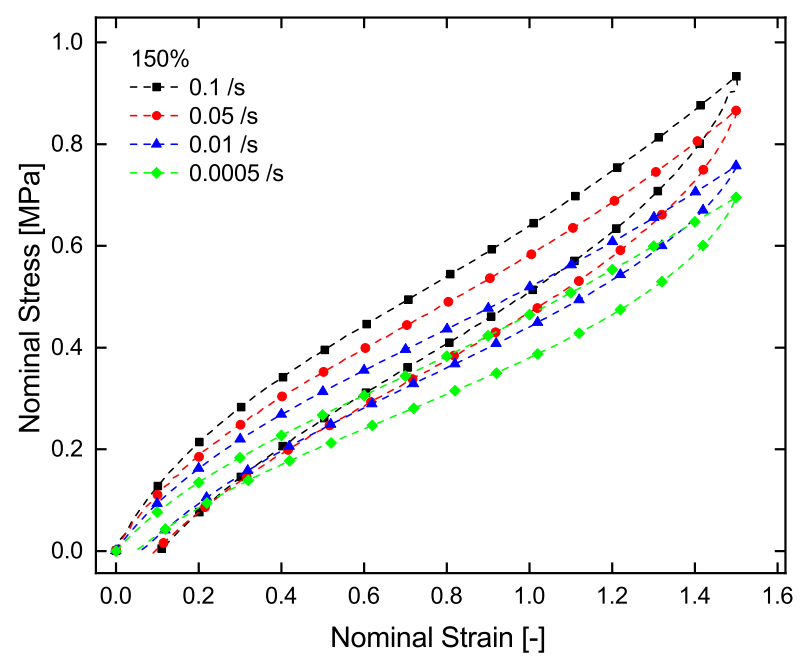

(a)

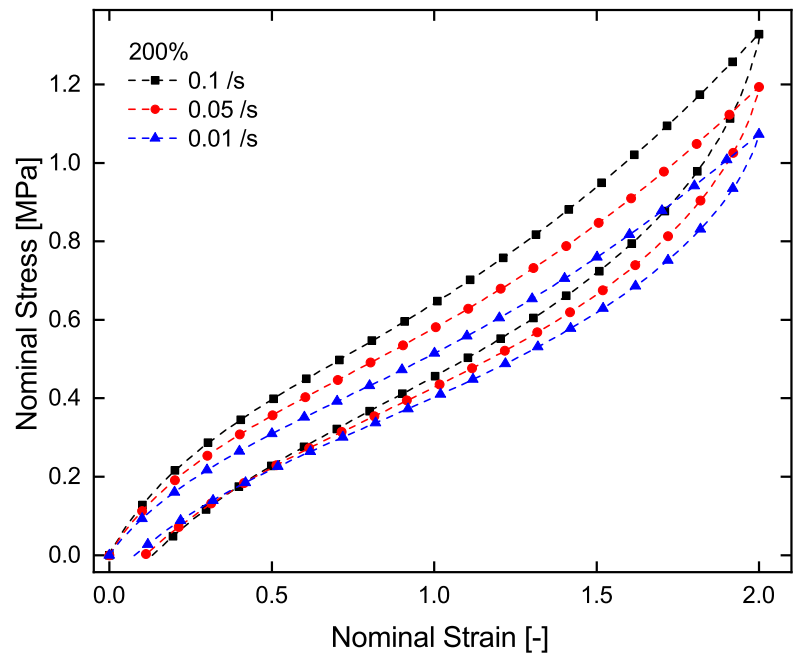

(b)

Figure 7. Loading-unloading cyclic experiments at four strain rates 0.1 /s, 0.05 /s, 0.01 /s, and 0.0005/s: (a) 150\% strain, (b) $200 \%$ strain. A significant strain rate-dependence and nonlinear stress-strain relation is illustrated by a hardening-softening-hardening behaviour within these strain ranges.

following cycles. In order to re-assure the existence of the Mullins effect, a second form of tests is also required. For this, after a full loading-unloading cycle, the sample is reloaded to a higher strain (keeping the strain rate same as in the first full cycle). In the second loading cycle, the actual strain will be larger than the already applied maximum strain in the first cycle. The stress response will be lower than the first cycle up to the maximum strain already applied. However, as soon as the sample crosses the maximum strain already applied in the first cycle, it goes along the same loading path of the first cycle. If this is the case, then the polymer exhibits Mullins effect. Both features are important characteristics of the Mullins effect [18, 44].

In this study, both categories of the stress-softening tests are performed on SIL30 polymer. In Fig. 5(a), experimental results of ten repeated cycles are presented for a sample with $100 \%$ strain. Fig. 5(a) depicts the stress-strain response of a primary loading path which clearly shows a nonlinear material behaviour for a virgin specimen. If we compare the first (primary) loading path with rest of the cycles, it is vivid that each reloading path in the second and the subsequent cycles experience significant stress reductions. Note that most of the stress reductions occur between the first and the second cycles. Moreover, after the third cycle, the differences between the previous cycles and an actual cycle minimise that eventually result in a stable loading-unloading dissipative loop. We further quantify the existence of Mullins effect with the help of cyclic tensile tests with increasing strains. Fig. 5(b), illustrates that if the actual strain becomes larger than the already applied maximum strain, the stress-strain response goes along the same loading path of the first cycle. Hence, after performing two different types of tests, it can be concluded that the 3D printed silicone polymer (SIL30) shows a significant stress-softening or Mullins effect. Therefore, before performing any viscoelastic experiments, three cycles of pre-treatments have been done on each virgin sample. Afterwards, all samples are used in the next day for actual thermo-viscoelastic experiments. 


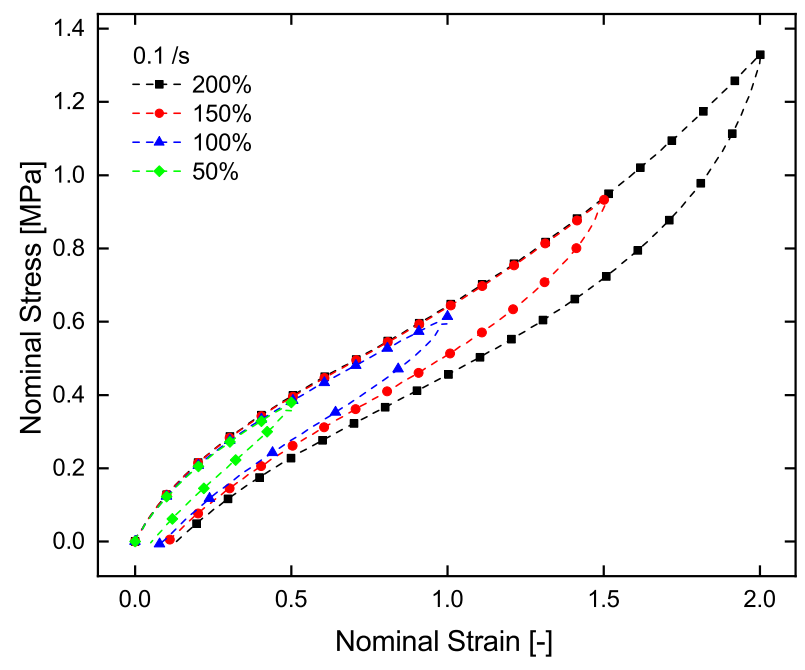

(a)

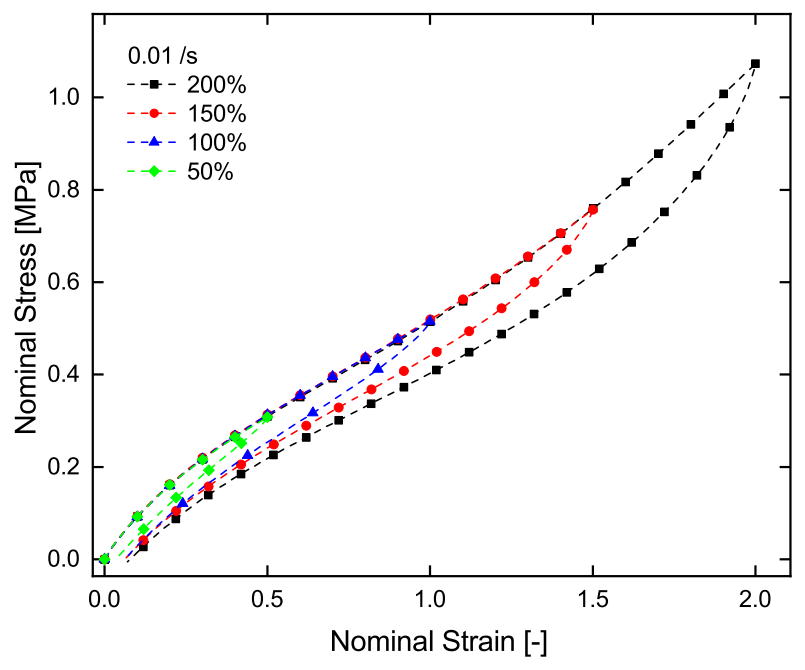

(b)

Figure 8. Loading-unloading cyclic experiments at four strain levels 50\%, 100\%, 150\%, and 200\%: (a) strain rate $0.1 / \mathrm{s}$, (b) strain rate 0.01 s. A higher strain rate results in a larger dissipative loop bounded by the loading-unloading paths.

\subsection{Loading-unloading cyclic behaviour}

One of the major characteristics of soft polymers is their dissipative behaviour. Majority devices made of soft polymeric materials experience a large number of loading-unloading cycles in their service lives. An unloading path of a polymer will not coincide its loading path that results in a loading-unloading dissipative loop. Moreover, stress-strain responses will significantly be varied with respect to how fast or how slow a deformation is applied to the material. For the viscoelastic characterization, at first loading-unloading cyclic tests are performed with respect to different strains, strain rates, and temperature profiles. In this study, based on the stretchability results presented in the previous section, four different strain levels, i.e., 50\%, $100 \%, 150 \%$, and 200\%; four different strain rates, i.e., $0.0005 / \mathrm{s}, 0.01 / \mathrm{s}, 0.05 / \mathrm{s}$, and $0.1 / \mathrm{s}$ are selected. These consist of (at least) total forty eight tests ( $=4$ strains $\mathrm{x} 4$ strain rates $\mathrm{x} 3$ repetitions) that are performed at room temperature $\left(20^{\circ} \mathrm{C}\right)$. For quantifying temperature-dependent behaviour, five different temperature profiles, i.e., $60^{\circ} \mathrm{C}, 40^{\circ} \mathrm{C}, 20^{\circ} \mathrm{C}, 0^{\circ} \mathrm{C}$, and $-20^{\circ} \mathrm{C}$ are selected in which strain rates of $0.1 / \mathrm{s}$ and $0.01 / \mathrm{s}$ and strains of $50 \%, 100 \%$, and $200 \%$ are chosen (total 72 tests $=4$ temperature profiles as the tests at $20^{\circ} \mathrm{C}$ are already performed $\mathrm{x} 3$ strains $\mathrm{x} 2$ strain rates $\mathrm{x} 3$ repetitions). Note that if a good reproducibility is not obtained, some of these tests are repeated up to five times.

\subsubsection{Results and discussions}

Results shown in Fig. 6 and Fig. 7 are room temperature $\left(20^{\circ} \mathrm{C}\right)$ data. Fig. 6(a) and Fig. 6(b) compare the loading-unloading curves with four different strain rates of 50\% and 100\% strains, respectively, wherein the stress-strain curves clearly depict a strain rate-dependence of the 3D printed silicone. Therein, a high strain rate results in a higher stress response and the vice-versa. Moreover, the curves demonstrate significant nonlinear stress-strain behaviour at room temperature, in which strain hardening occurs initially and then strain softening exists up to $100 \%$ strain which follows by a strain hardening again. Again, if someone compares Fig. 6 and Fig. 7 of 50\%, 100\%, 150\%, and 200\% strains, it can be seen that the nonlinearity of the curves even enhances further with higher strains. After $100 \%$ strain, the strain-strain curves show upturn trend which is, at 200\% strain clearly resembling an 'S-shape' curve, a typically stress-strain behaviour observed 


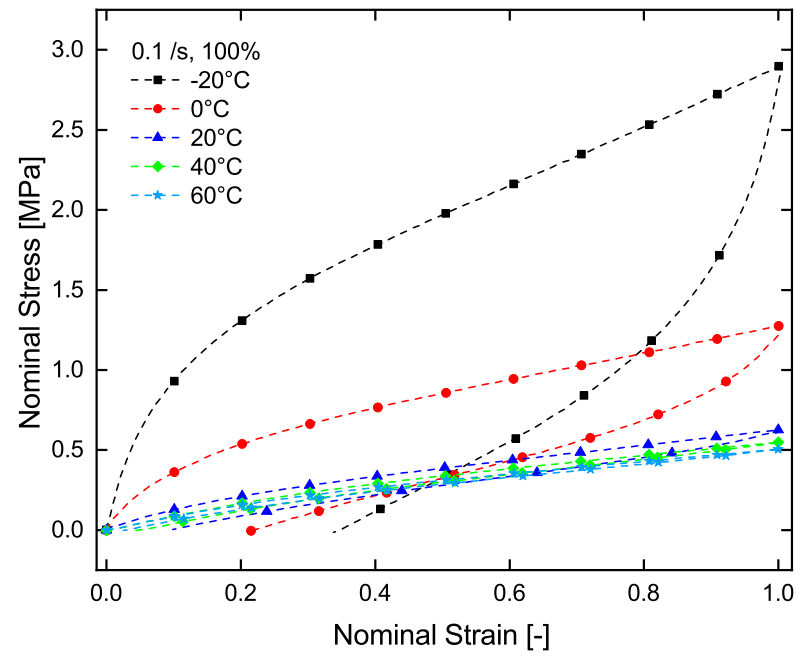

(a)

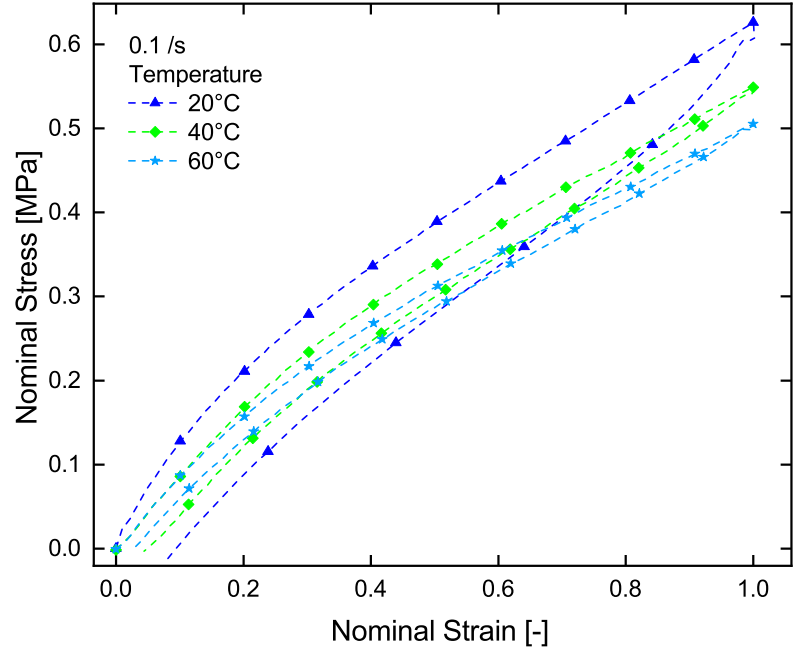

(b)

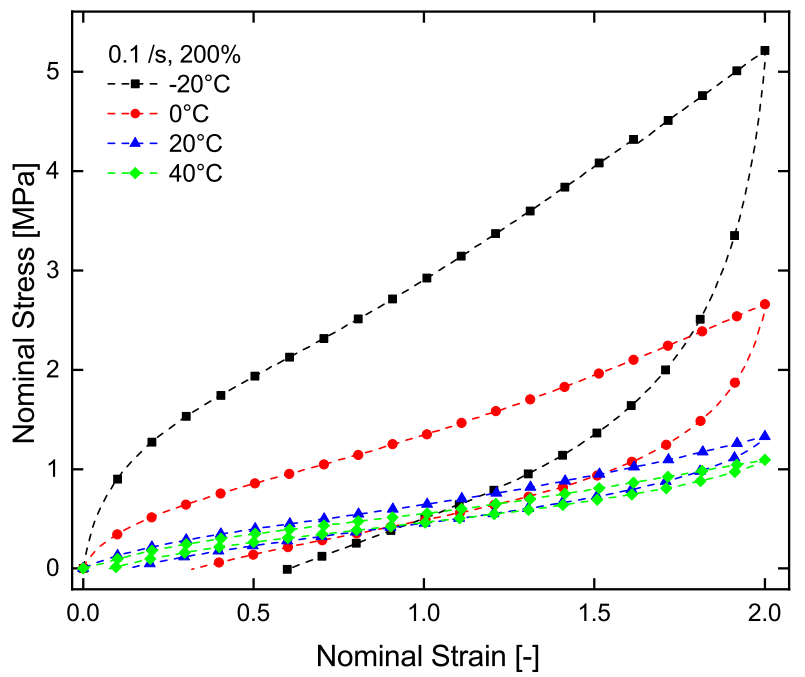

(c)

Figure 9. Temperature-dependent cyclic test results at strain rate $0.1 / \mathrm{s}$ and at temperature profiles $-20^{\circ} \mathrm{C}, 0^{\circ} \mathrm{C}, 20^{\circ} \mathrm{C}, 40^{\circ} \mathrm{C}$, and $60^{\circ} \mathrm{C}$ : (a) $100 \%$ strain, (b) a close-up view of (a), (c) $200 \%$ strain. The influences of temperature profile are massive in negative temperature regime in contrast to its influences in positive regime. 


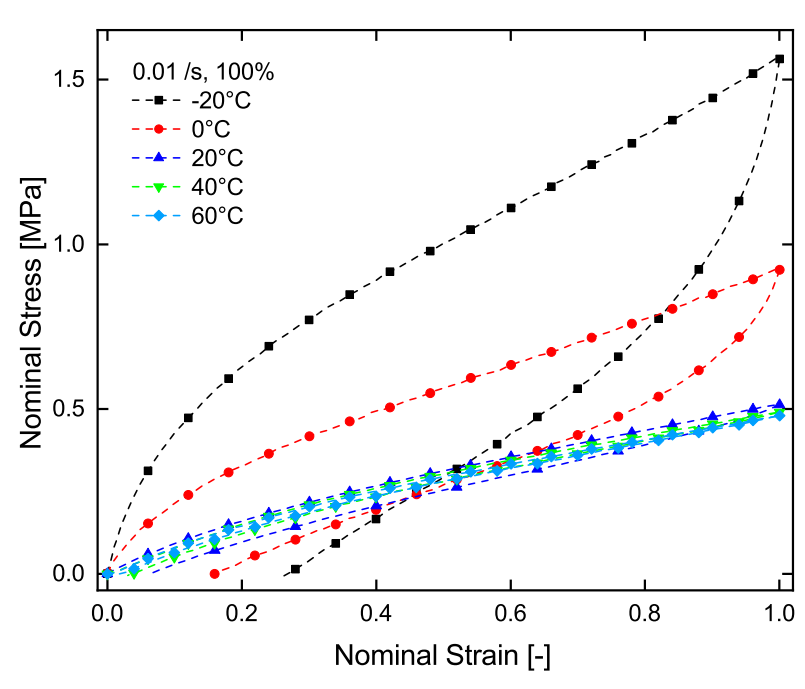

(a)

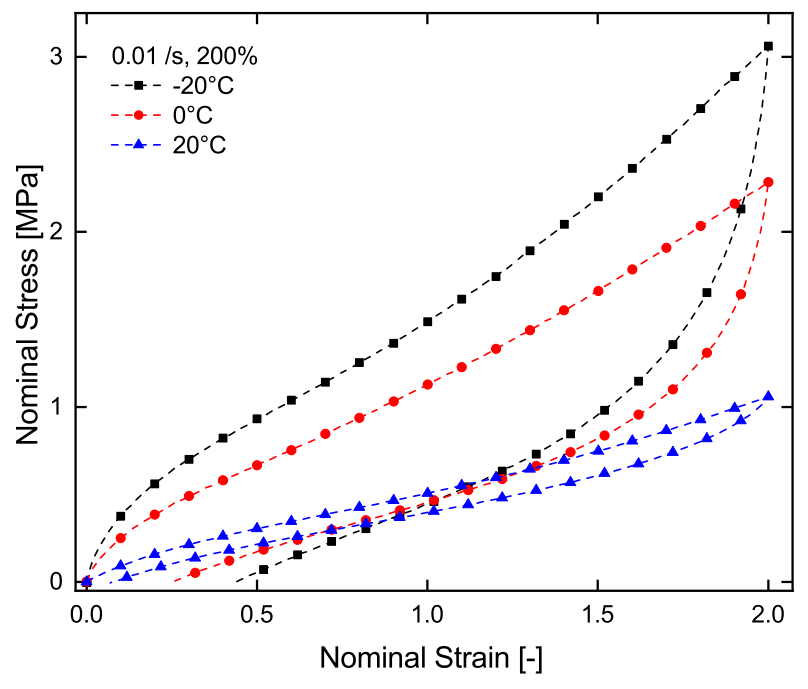

(b)

Figure 10. Temperature-dependent cyclic test data at strain rate $0.01 / \mathrm{s}$ and at temperature profiles $-20^{\circ} \mathrm{C}, 0^{\circ} \mathrm{C}, 20^{\circ} \mathrm{C}, 40^{\circ} \mathrm{C}$, and $60^{\circ} \mathrm{C}$ : (a) $100 \%$ strain, (b) $200 \%$ strain.

in highly stretchable soft elastomers. Furthermore, the dissipative loops bounded by loading-unloading paths at different strains increase with the increasing strain rates, cf. Fig. 8. Note that some classical silicone polymers such as Ecoflex, Sylgard 184, RTV 141, RTV 3428, B452, and Sil8800 do not show any major strain rate-dependent behaviour at room temperature. In contrast, the 3D printed silicone (SIL30) shows a significant strain rate-dependence which might be good for its applications in damping.

In many applications, silicone polymers are exposed to variations of temperature profiles. Hence, it is imperative to have a comprehensive thermo-mechanical study of any polymer prior to its intended applications. For investigating the influences of temperature, few classical silicones have been studied over the years that were documented in the literature, see $[6,35,47,53]$. Therein most of the silicones hardly show any temperature-sensitive behaviour. However, the present study reveals a high temperature-sensitivity of silicone SIL30, in which a higher temperature reduces its stiffness significantly. On the contrary, the polymer becomes stiffer at a lower temperature resulting in a higher modulus. According to a manual supplied by Carbon3D [12], the polymer has a glass transition temperature $\left(T_{g}\right)$ of $8^{\circ} \mathrm{C}$. For the temperature-dependent viscoelastic study, five different temperature profiles are selected within the range of $-20^{\circ} \mathrm{C}$ to $60^{\circ} \mathrm{C}$, in which two different strain rates, $0.1 / \mathrm{s}$ and $0.01 / \mathrm{s}$, are applied. Fig. 9 and Fig. 10 indicate that for all temperature profiles under investigation, a lower temperature results in an enlarge loading-unloading hysteresis loop and a larger residual strain. This means, the material becomes highly viscous below the room temperature which is manifested by the large dissipative loops for both $0.1 / \mathrm{s}$ and $0.01 / \mathrm{s}$ strain rates. Furthermore, at the same strain rate and strain, the nonlinearity in the stress-strain relationship is becoming even stronger with the decreasing temperature. These features are more vivid when temperature-dependent residual strains and peak stresses are plotted against temperature profiles, see Fig. 11. Especially, abrupt changes in residual strains and peak stresses can be observed below the glass transition temperature $\left(T_{g}=8^{\circ} \mathrm{C}\right)$ of the polymer.

Note that the silicone polymer is less temperature-sensitive above the room temperature due to the fact that it 


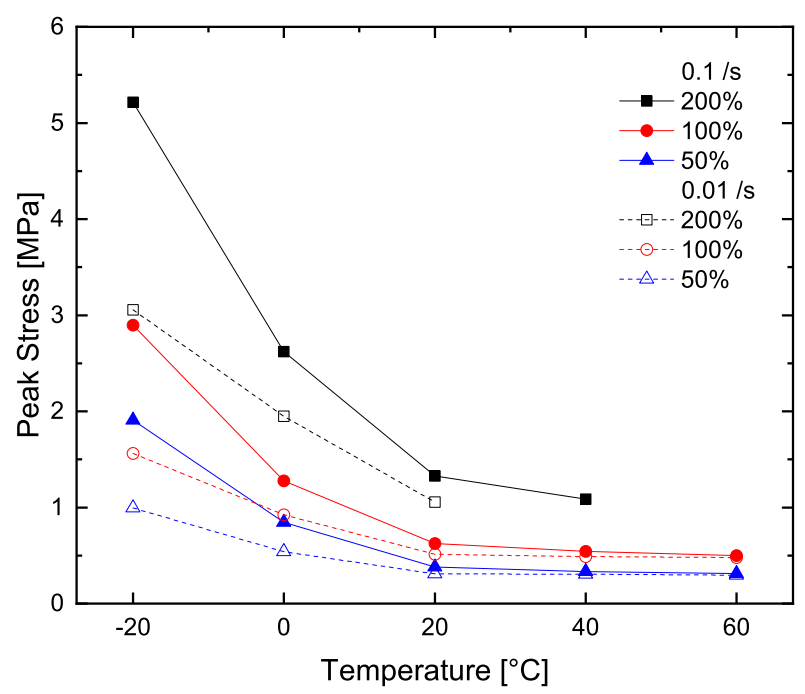

(a)

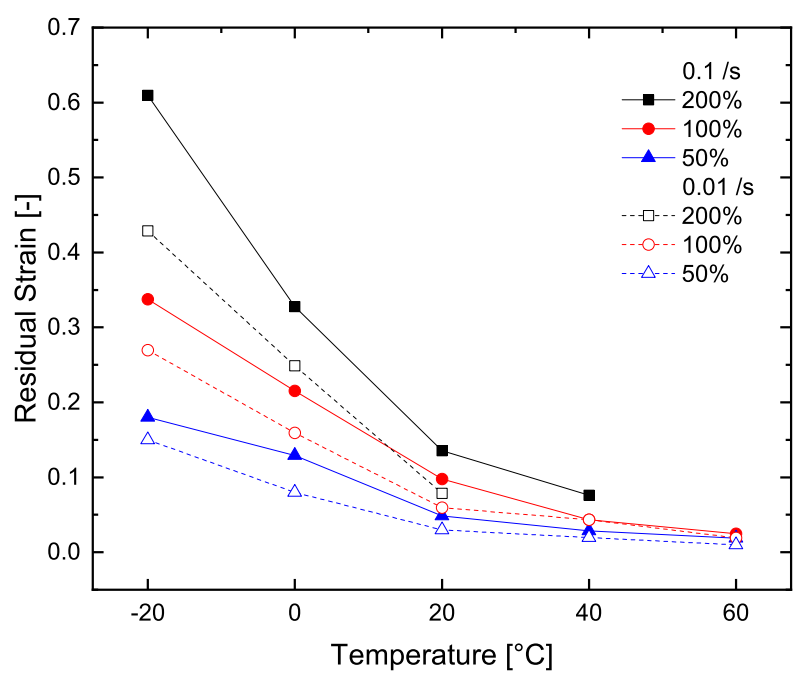

(b)

Figure 11. Influences of temperature on residual strains and peak stress values, (a) Variations of peak stresses with temperature profiles, (b) Sharp changes of the residual strains with respect to temperature variations.

becomes entropic elastic in these temperature ranges. Furthermore, if Fig. 9(a) and Fig. 9(b) are compared, it is clear that the nonlinearity modes in the strain-strain relationship are distinct in low temperature in contrast to one above the room temperature. For instance, in Fig. 9(a), the stress-strain curve at $-20^{\circ} \mathrm{C}$ becomes nonlinear with an initial strain hardening followed by a softening, whereby a similar trend is observed in the unloading path. In contrast, as shown in Fig. 9(b), for all temperature profiles above $20^{\circ} \mathrm{C}$, the loadingunloading curves follow almost a linear pattern with a minor change. Such a large scale dissipative cycles in the negative temperature profile is due to the fact that in this region the material obtains the transition from a rubbery phase to a glassy phase.

\subsection{Relaxation behaviour: Single-step and multi-step tests}

Stress relaxation is a classical phenomenon in viscoelastic soft polymers. Many devices made of soft polymeric materials are exposed to strains either in one or in several steps with multiple increments [14]. In both cases, polymeric materials experience stress relaxations. Hence, on the one hand, to assess materials' stress relaxation behaviour at single-and multiple-strains, both types of relaxation experiments are imperative. On the other hand, the two types of experiments will simply help to determine the equilibrium stress of a polymer. For a relaxation test, a specimen is stretched at a particular speed of the machine and is held at a desired strain. Afterwards, if we wait for several hours, the sample will reach to a stress that does not change significantly further with respect to time. This is called the equilibrium stress. For single-step relaxation tests, $25 \%, 50 \%, 75 \%, 100 \%, 125 \%$, and $150 \%$ strains are applied at room temperature that result in eighteen tests ( 6 strains $\mathrm{x} 3$ repetitions). Moreover, three additional temperatures $-20^{\circ} \mathrm{C}, 0^{\circ} \mathrm{C}$ and $40^{\circ} \mathrm{C}$ for $50 \%$ deformations and two more temperature profiles, i.e., $-20^{\circ} \mathrm{C}$ and $0^{\circ} \mathrm{C}$, are added in the case of $100 \%$ deformations to quantity the influences of temperature on the relaxation mechanisms. Note that all multistep relaxation experiments are performed at room temperature. 


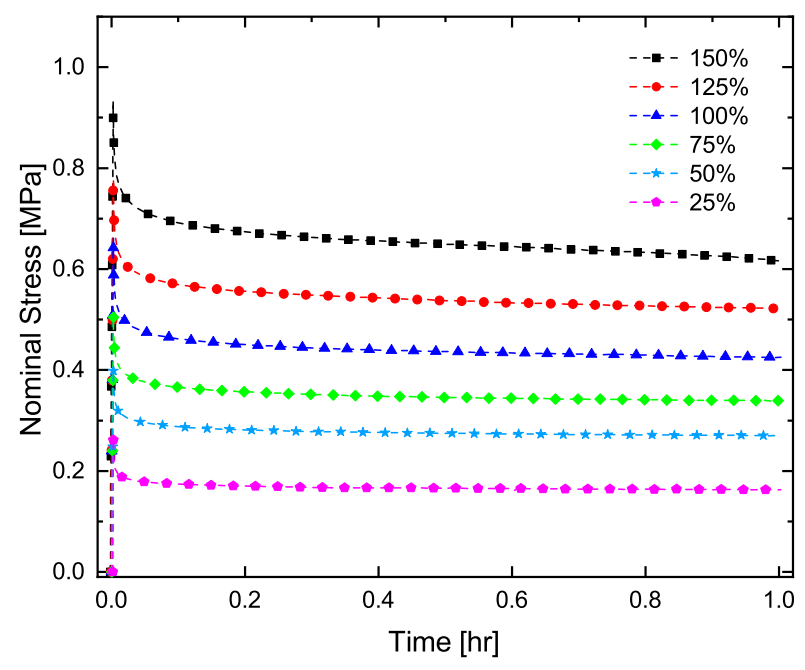

(a)

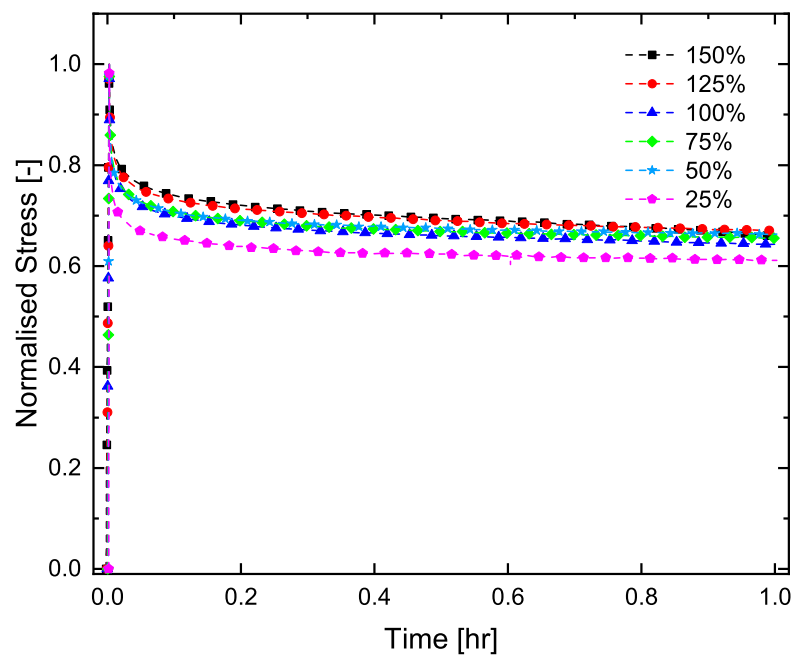

(b)

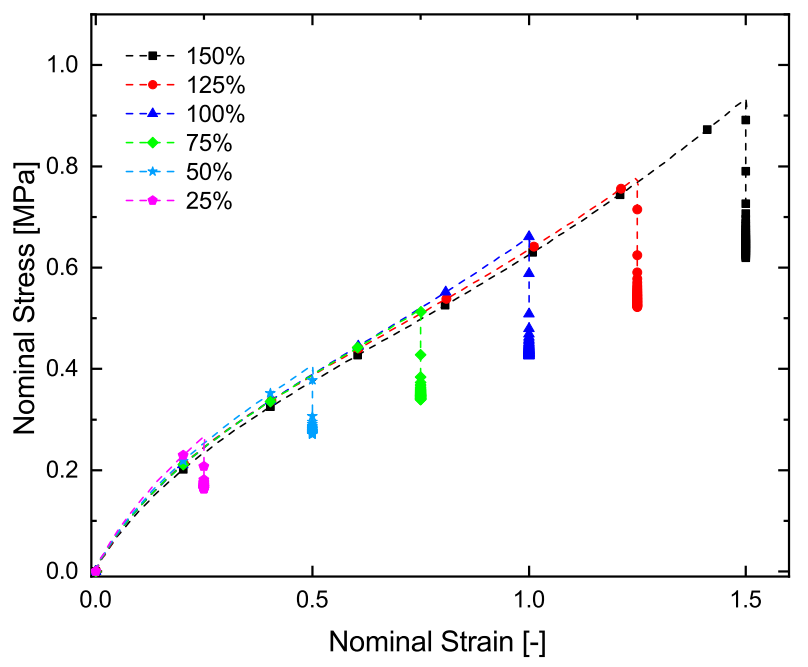

(c)

Figure 12. Single-step relaxation tests from $25 \%$ to $150 \%$ with a $25 \%$ strain interval. (a) Stress-time curves, (b) normalised stresstime curves obtained by dividing the actual stresses with the peak stresses, (c) stress-strain curves. The relaxation time is increasing with the increase of strains. 


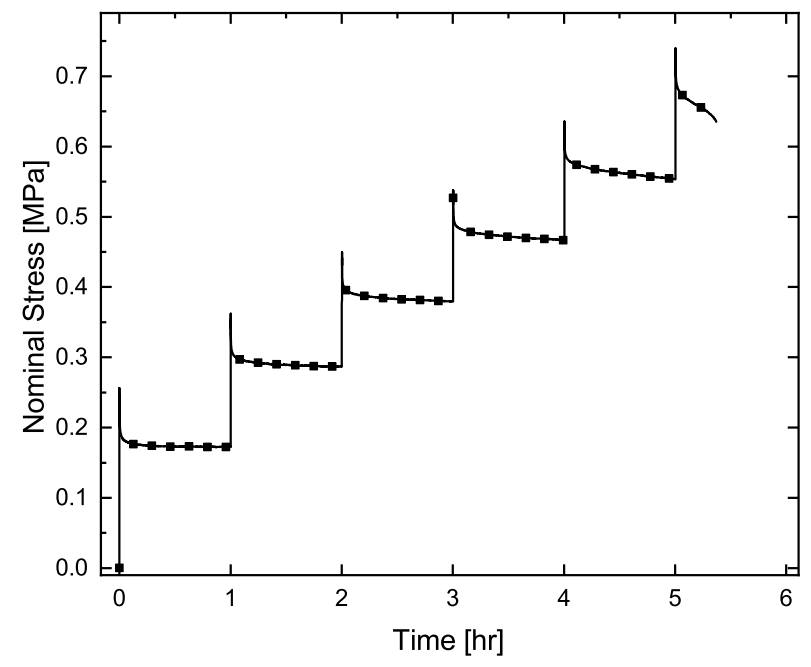

(a)

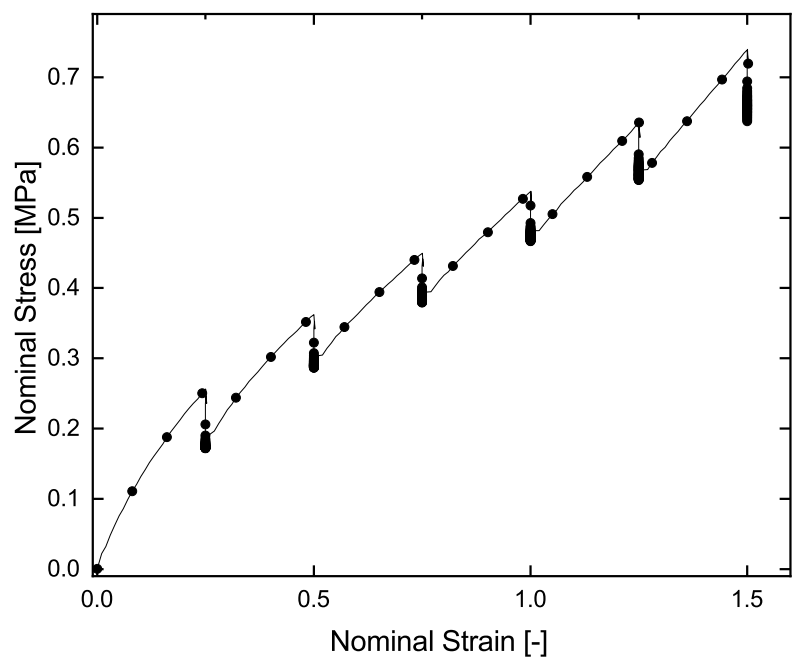

(b)

Figure 13. Multi-step relaxation test data at strain steps from $25 \%$ to $150 \%$ with a $25 \%$ strain interval. (a) Stress history curves, (b) stress-strain curves.

\subsubsection{Results and discussions}

In the absence of any available data demonstrating the relaxation behaviour of SIL30, a sample is allowed to relax for several hours. However, it is observed that almost all of the so-called overstress (total stress minus equilibrium stress) disappear within the first one hour period, cf. Fig. 12. Hence, for the rest of the stress relaxation experiments, an hour holding time is selected both for the single-step and the multi-step (in each step strain) relaxation experiments. The stress at the end of an hour holding time is considered as the equilibrium stress. For single-step relaxation tests, specimens are elongated starting from $25 \%$ to $150 \%$ strain with a $25 \%$ interval. The material is unable to sustain any strain beyond $150 \%$ for a longer time in single-and multi-step relaxation tests. In order to understand the relaxation phenomena, we can compare the behaviour of the material at two different deformations, i.e., $25 \%$ and $150 \%$. For tests at $150 \%$ deformation, after 20 seconds of the relaxation, the normalised actual stress becomes 0.862 (peak stress $=1.0$ ) which reduces to $0.802,0.694$, and 0.658 , respectively, after $1 \mathrm{~min}, 30 \mathrm{mins}$, and 1 hour of the relaxation. In contrast, for $25 \%$ deformation, after 20 seconds, the normalised actual stress becomes 0.769 which reduces to $0.703,0.623$, and 0.611 , respectively, after $1 \mathrm{~min}, 30 \mathrm{mins}$, and 1 hour of the relaxation. From the analyses, several conclusions can be drawn for the 3D printed SIL30. Most of the overstresses are depleted within a few seconds of the holding time. For instance, $20 \%$ overstress disappears after 20 seconds of the relaxation for $150 \%$ deformation, while a $24 \%$ reduction in the stress for samples stretched at $25 \%$. That means, a relaxation test conducted at a higher strain will take a longer time to reach an equilibrium stress. This will be more vivid when normalised stresses obtained by dividing the actual stresses with the peak stresses are plotted against the relaxation time as in Fig. 12(b). It further reveals the strain-dependence of the relaxation behaviour. It also substantiates our assumption that one hour holding time is enough to obtain an equilibrium stress as the differences in overstresses between half an hour and one hour are very minimal (1\%-3\%). Now, if we connect all equilibrium stresses, the basic elasticity data of the material will be obtained.

In an effort to quantify the material's relaxation behaviour at multiple strains, few more relaxation tests are performed, in which incremental strains are applied. Another aim of these tests is to verify the equilibrium 


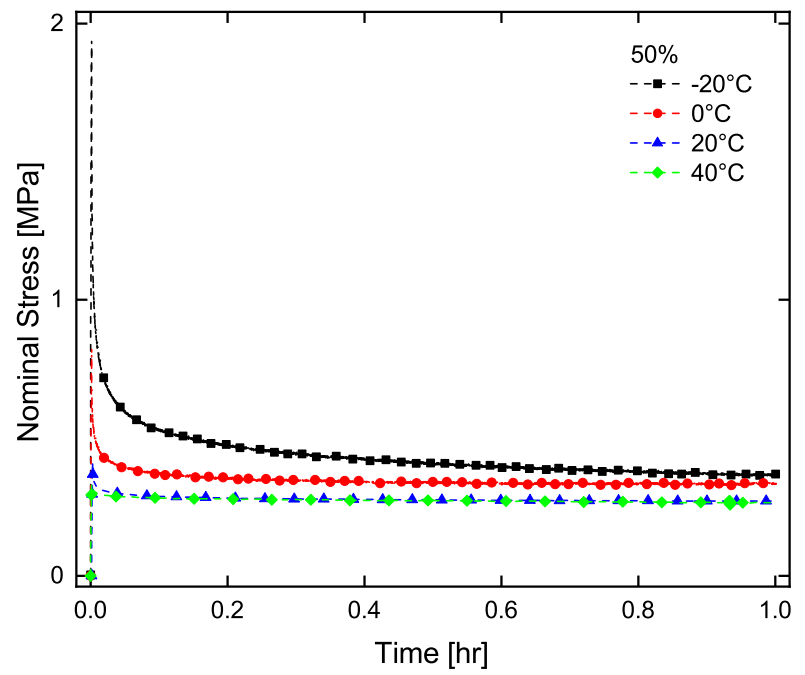

(a)

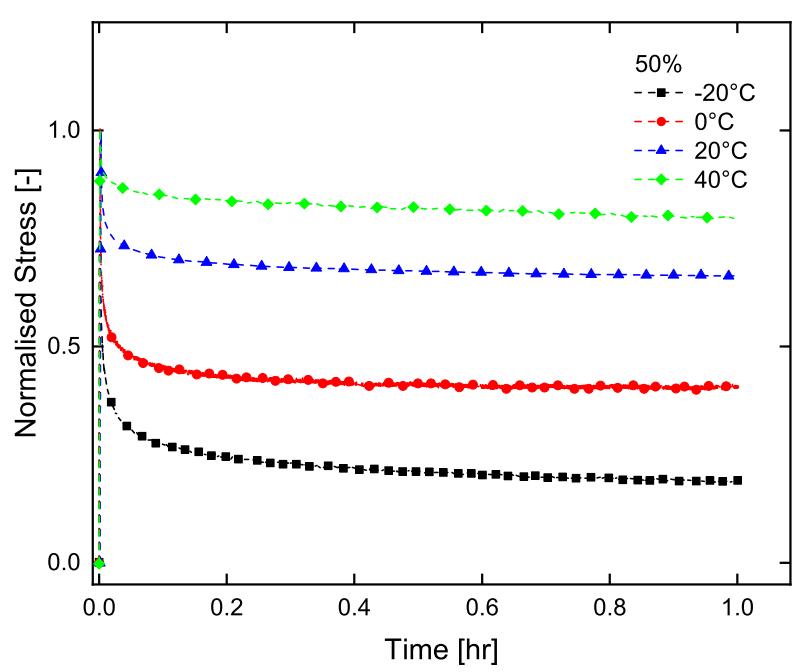

(b)

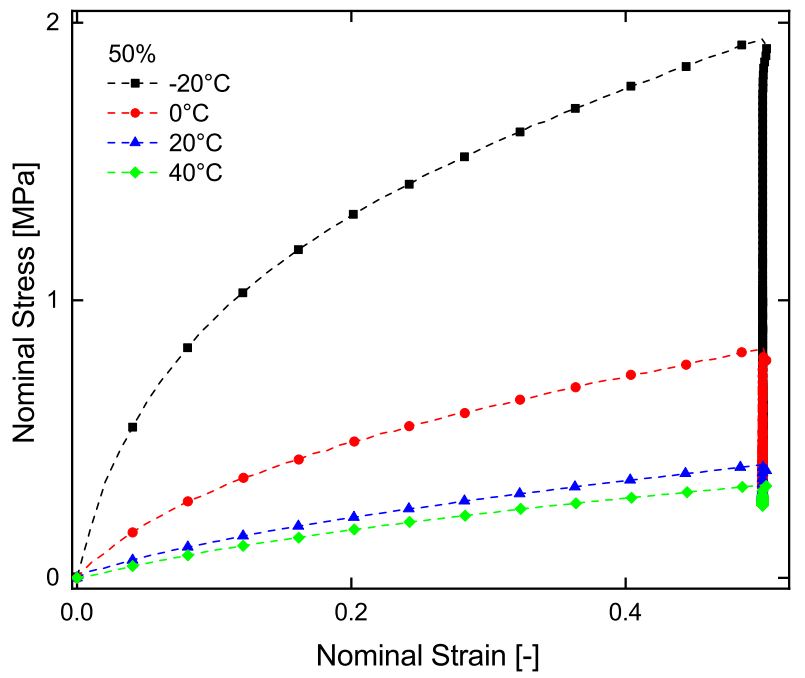

(c)

Figure 14. Temperature dependent relaxation test results at $50 \%$ strain and at temperature profiles $-20^{\circ} \mathrm{C}, 0^{\circ} \mathrm{C}, 20^{\circ} \mathrm{C}$, and $40^{\circ} \mathrm{C}$. (a) Stress history curves, (b) normalised stress-time curves obtained by dividing the actual stresses with the peak stresses, (c) stress-strain curves. 
stresses of the polymer obtained by the single-step relaxation tests performed earlier. In comparison to the step-wise relaxation tests, for the multi-step relaxation experiments, a specimen is elongated in several consecutive steps and it is allowed to relax in each step. In addition to other usages of the multi-step relaxation tests, with the help of one relaxation test set-up, several equilibrium stresses at few strain intervals can smoothly be obtained. In this study, to observe relaxation behaviour at multiple strains, step strains are applied at intervals of $25 \%, 50 \%, 75 \%, 100 \%, 125 \%$, and $150 \%$ strains. Further efforts are put to increase the strains beyond $150 \%$ for the 3D printed silicone. However, the material breaks after six consecutive step strains of six hours (total) time which is vivid in Fig. 13(a). Fig. 13 shows that for a particular strain, relaxed stress (equilibrium stress) data from a single-step relaxation test (Fig. 12(c)) and a multi-step relaxation tests (Fig. 13(b)) closely share the same history. In summary, the 3D printed polymer shows significant stress relaxation phenomena which can be diminished with an hour of the holding time. The relaxed stresses obtained from the two different types of experiments will be used to develop a thermo-viscoelastic model in Section 3. Note that all multi-step relaxation tests are conducted at room temperature $\left(20^{\circ} \mathrm{C}\right)$.

For understanding the effects of temperature on relaxation mechanisms, a couple of single-step relaxation tests are performed within the temperature chamber. For this, single-step relaxation experiments at $50 \%$ and $100 \%$ strains are selected with four different temperature profiles, i.e., $-20^{\circ} \mathrm{C}, 0^{\circ} \mathrm{C}, 20^{\circ} \mathrm{C}$, and $40^{\circ} \mathrm{C}$. One of the aims of these tests (Fig. 14(a)), at first, is to assess the influences of temperature on the equilibrium stresses of the material. Fig. 14 and Fig. 15 illustrate single-step relaxation results of 50\% and $100 \%$ step strains, respectively, for four different temperature profiles. Owing to thermal influences below the room temperature, total (peak) stresses are increasing dramatically in contrast to the total stress above the room temperature. However, after stress relaxations in both cases, there are no significant differences in equilibrium stresses. For instance, if we compare total stresses for experiments conducted at $-20^{\circ} \mathrm{C}(2.0 \mathrm{MPa})$ and $20^{\circ} \mathrm{C}(0.4 \mathrm{MPa})$ up to $50 \%$ strain, the former is almost five times larger than the latter, cf. Fig. 14(c). However, their equilibrium stresses are almost identical. Noteworthy to mention here that at higher strains, the stress relaxation requires more time to obtain the equilibrium stresses. Therefore, a single-step relaxation experiment conducted under $-20^{\circ} \mathrm{C}$ requires more relaxation time than the one at $20^{\circ} \mathrm{C}$ up to $100 \%$ strain, see Fig. 15. Such an assertion can also be illustrated with the help of normalised stress versus time curves as in Fig. 14(b) and Fig. 15(b). Hence, despite a significant difference in relaxed stresses of the two tests, it can be assumed that after a sufficient time of relaxation, the actual stress of the former will reduce to a closer value of the latter. In summary, it can be assumed that temperature has significantly less influence on the equilibrium (elastic) stresses than on the non-equilibrium (viscous) stresses.

\section{A constitutive model for thermo-viscoelastic polymers}

\subsection{Kinematics}

One of the classical approaches in modelling viscoelasticity at finite strain is due to a multiplicative deformation of the deformation gradient $(\boldsymbol{F})$, where it is decomposed into an elastic and a viscous parts as

$$
\boldsymbol{F}=\boldsymbol{F}_{e}^{j} \boldsymbol{F}_{v}^{j}, \quad j=1, \cdots, N .
$$

The aforementioned decomposition resembles the spring-dashpot analogy in small-strain viscoelasticity, where $N$ counts the number of Maxwell elements that represent the possibility of multiple viscous mechanisms in polymeric materials, see Reese and Govindjee [46], Liao et al. [34]. The decomposition of the deformation gradient yields few more strain tensors as $\boldsymbol{C}=\boldsymbol{F}^{T} \boldsymbol{F}, \boldsymbol{C}_{v}^{j}=\boldsymbol{F}_{v}^{j, T} \boldsymbol{F}_{v}^{j}$, and $\boldsymbol{C}_{e}^{j}=\boldsymbol{F}_{e}^{j, T} \boldsymbol{F}_{e}^{j}=$ 


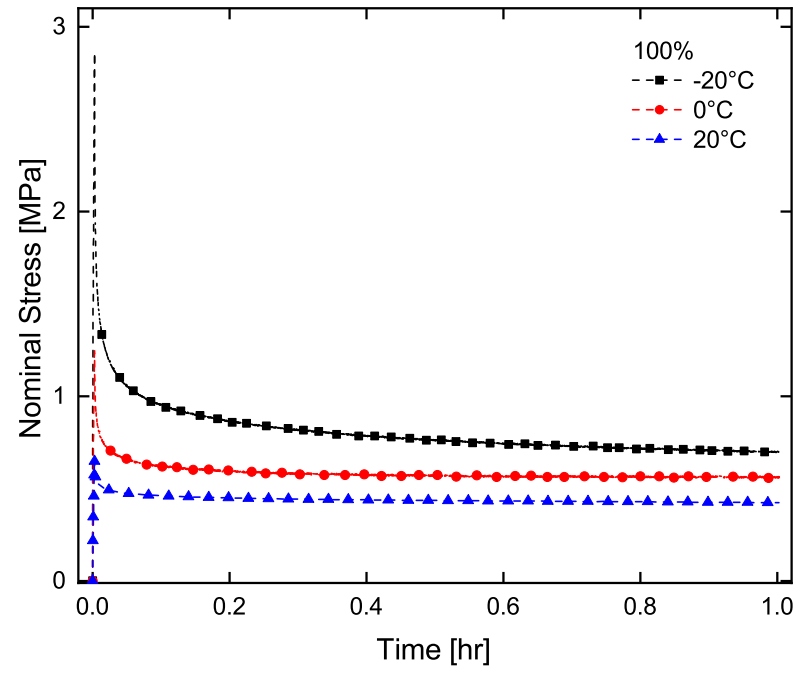

(a)

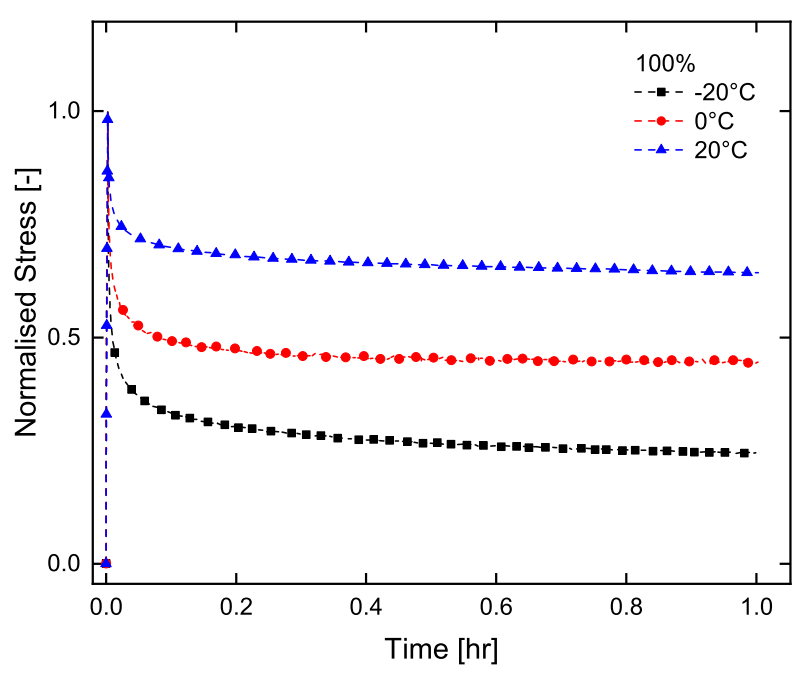

(b)

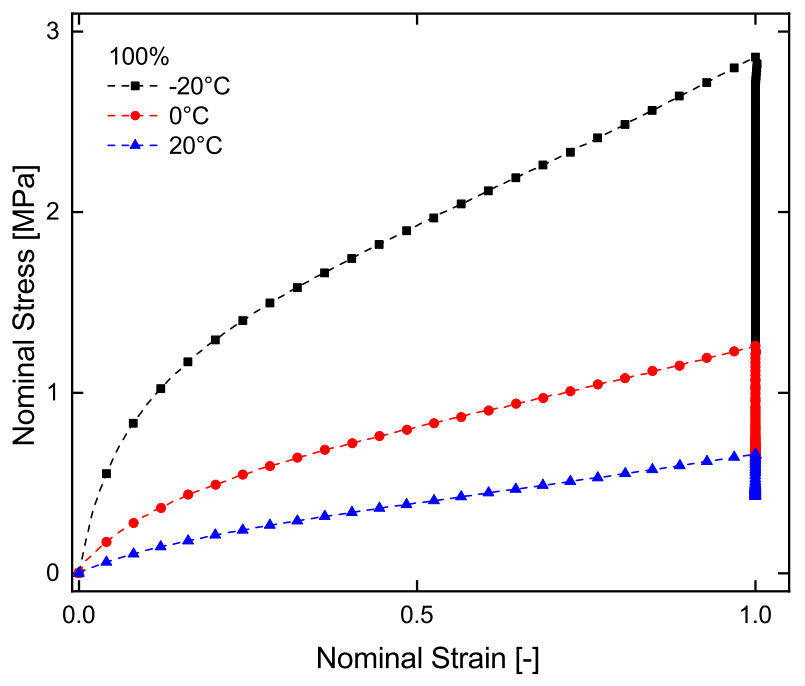

(c)

Figure 15 . Temperature-dependent relaxation test data at $100 \%$ strain and at temperatures $-20^{\circ} \mathrm{C}, 0^{\circ} \mathrm{C}$, and $20^{\circ} \mathrm{C}$. (a) Stress history curves, (b) normalised stress-time curves obtained by dividing the actual stresses with the peak stresses, (c) stress-strain curves. 
$\boldsymbol{F}_{v}^{j,-T} \boldsymbol{C} \boldsymbol{F}_{v}^{j,-1}$. Note that, for a generic setting, the deformation gradient needs to be decomposed further, i.e., $\boldsymbol{F}=\boldsymbol{F}_{e}^{j} \boldsymbol{F}_{v}^{j} \boldsymbol{F}_{\Theta}$ to capture the volumetric effects of the temperature field. However, Section 2 presents a plethora of experimental data that are obtained from homogeneous tensile tests. In modelling approaches, rubber-like polymers are widely considered as incompressible materials [3, 9]. Hence, the contribution from $\boldsymbol{F}_{\Theta}$ will be unity, see Mehnert et al. [41] for details.

\subsection{Stress formulations}

polymeric material is ideally decomposed into an elastic and a viscous parts as

$$
\Psi=\Psi_{e}(\boldsymbol{C}, \Theta)+\Psi_{v}\left(\boldsymbol{C}, \boldsymbol{C}_{e}, \Theta\right)=\Psi_{e}(\boldsymbol{C}, \Theta)+\sum_{j=1}^{N} \Psi_{v}^{j}\left(\boldsymbol{C}, \boldsymbol{C}_{e}^{j}, \Theta\right)
$$

where, $\Theta$ is the actual temperature. Note that the number of Maxwell elements $N$, required for a particular polymer, depends the degree of non-linearity of the experimental data. In order to obtain relevant relations for the stress tensors, we have to depart from the second law of thermodynamics in the form of ClausiusDuhem inequality which, in the case of thermo-viscoelastic material, is

$$
-\dot{\Psi}-\zeta \dot{\Theta}-\frac{\boldsymbol{Q}}{\Theta} \cdot \operatorname{Grad}_{\boldsymbol{X}} \Theta+\boldsymbol{S}: \frac{1}{2} \dot{\boldsymbol{C}}+p \boldsymbol{C}^{-1}: \frac{1}{2} \dot{\boldsymbol{C}} \geq 0
$$

where, $\zeta$ is the entropy, $Q$ is the heat flux vector, $p$ is a Lagrange multiplier and the last term of the above equation is due to the incompressibility constraint for rubber-like materials, see Mehnert et al. [41]. Following Coleman-Noll argumentation [8], an expression of the stress yields

$$
\boldsymbol{S}=-p \boldsymbol{C}^{-1}+2 \frac{\partial \Psi_{e}}{\partial \boldsymbol{C}}+\sum_{j=1}^{N} 2 \boldsymbol{F}_{v}^{j,-1} \frac{\partial \Psi_{v}^{j}}{\partial \boldsymbol{C}_{e}^{j}} \boldsymbol{F}_{v}^{j,-T}=-p \boldsymbol{C}^{-1}+\boldsymbol{S}_{e}+\sum_{j=1}^{N} \boldsymbol{S}_{v}^{j} .
$$

\subsection{A specialised thermo-viscoelastic energy function}

In order to complete the expressions for two parts of the stress tensors in Eq. 4, an appropriate choice for the energy function is required that depends on strain, internal variable(s), and temperature field. Furthermore, the strain energy needs to be scaled with some functions that depend on temperature profile only. Very recently, Mehnert et al.[41], Liao et al.[34], Behnke et al. [5] formulated thermo-viscoelastic energy functions following the relevant laws of thermodynamics. Such a thermo-viscoelastic energy function can be presented as

$$
\Psi=\left[\frac{\Theta}{\Theta_{0}}+g_{e}(\Theta)\right] \Psi_{e}(\boldsymbol{C})+\sum_{j=1}^{N}\left[\frac{\Theta}{\Theta_{0}}+g_{v}^{j}(\Theta)\right] \Psi_{v}\left(\boldsymbol{C}, \boldsymbol{C}_{e}^{j}\right)-c_{0}\left[\Theta-\Theta_{0}-\Theta \ln \left(\frac{\Theta}{\Theta_{0}}\right)\right]
$$

where, $g_{e}, g_{v}^{j}$ are temperature-dependent scaling functions that are related to time-independent hyperelastic and time-sensitive viscous strain energies, respectively. In Eq. 5, $\Theta_{0}$ and $c_{0}$ are the reference temperature and a specific heat capacity at a constant temperature, respectively. Appropriate sets of experimental data will be required to identify these scaling functions. More details will be followed in Section 4. For complete expressions of the two stress tensors $\left(\boldsymbol{S}_{e}\right.$ and $\boldsymbol{S}_{v}$ ), two strain and temperature-dependent energy 
functions are required. For the elastic part of the energy function, a three-parameter Carroll model is chosen since it outperforms in predicting hyperelastic data of many soft polymeric materials, see [9, 22, 52]. A temperature-dependent elastic strain energy can be obtained by multiplying the strain energy devised for room temperature with a function $g_{e}$ as

$$
\Psi_{e}(\boldsymbol{C}, \Theta)=\left[\frac{\Theta}{\Theta_{0}}+g_{e}(\Theta)\right]\left[a I_{1}+b I_{1}^{4}+c\left[I_{2}\right]^{\frac{1}{2}}\right]
$$

In the aforementioned equation, $a, b, c$ are material constants and $I_{1}, I_{2}$ are the first and second invariants, respectively, of $\boldsymbol{C}$. From equation (6) and as per the definition of the elastic stress in (4), the elastic portion of the stress tensor can be determined as

$$
\boldsymbol{S}_{e}=2 \frac{\partial \Psi_{e}}{\partial \boldsymbol{C}}=\left[\frac{\Theta}{\Theta_{0}}+g_{e}(\Theta)\right]\left[\left[2 a+8 b I_{1}^{3}\right] \boldsymbol{I}+c\left[I_{1} \boldsymbol{I}-\boldsymbol{C}\right] I_{2}^{-\frac{1}{2}}\right], \quad \boldsymbol{I} \equiv \delta_{i j}
$$

Similar to the hyperelastic part of the energy function, we also take Carroll model for the viscous part that is related to spring(s) of the Maxwell element(s). Note that experimental data illustrated in Section 2 are rather complex in terms of non-linear stress-strain relationship. Hence, several Maxwell elements might be required in the non-equilibrium energy functions. In this case, a generic Carroll-type viscous energy function will be

$$
\Psi_{v}\left(\boldsymbol{C}, \boldsymbol{C}_{e}, \Theta\right)=\sum_{j=1}^{N} \Psi_{v}^{j}=\sum_{j=1}^{N}\left[\frac{\Theta}{\Theta_{0}}+g_{v}^{j}(\Theta)\right]\left[a^{j} I_{1 e}^{j}+b^{j}\left[I_{1 e}^{j}\right]^{4}+c^{j}\left[I_{2 e}^{j}\right]^{\frac{1}{2}}\right]
$$

where, $a^{j}, b^{j}, c^{j}$ are viscous material parameters. Note that $I_{1 e}^{j}$ and $I_{2 e}^{j}$ are the first and the second invariants, respectively, of $\boldsymbol{C}_{e}^{j}$, where $\boldsymbol{C}_{e}^{j}=\boldsymbol{F}_{v}^{j,-T} \boldsymbol{C F}_{v}^{j,-1}$. The remaining part of the total stress $(\boldsymbol{S})$ is the nonequilibrium viscous stress which can be expressed as

$$
\begin{aligned}
\boldsymbol{S}_{v}=2 \frac{\partial \Psi_{v}}{\partial \boldsymbol{C}} & =\sum_{j=1}^{N} 2 \boldsymbol{F}_{v}^{j,-1} \frac{\partial \Psi_{v}^{j}}{\partial \boldsymbol{C}_{e}^{j}} \boldsymbol{F}_{v}^{j,-T} \\
& =\sum_{j=1}^{N}\left[\frac{\Theta}{\Theta_{0}}+g_{v}^{j}(\Theta)\right] \boldsymbol{F}_{v}^{j,-1}\left[\left[\left[2 a^{j}+8 b^{j}\left[I_{1 e}^{j}\right]^{3}\right] \boldsymbol{I}+c^{j}\left[I_{1 e}^{j} \boldsymbol{I}-\boldsymbol{C}_{e}\right]\left[I_{2 e}^{j}\right]^{-\frac{1}{2}}\right]\right] \boldsymbol{F}_{v}^{j,-T}(9)
\end{aligned}
$$

Up to now, all relevant stress tensors are defined and calculated. In order to complete the thermo-viscoelastic model, an evolution equation is required to obtain an update of $\boldsymbol{C}_{v}$, which is widely termed as an internal variable. One of the classical finite strain linear evolution equation is as follows

$$
\dot{\boldsymbol{C}}_{v}^{j}=\frac{1}{\tau^{j}}\left[\boldsymbol{C}-\boldsymbol{C}_{v}^{j}\right], \quad j=1, \cdots, N,
$$

where, $\tau^{j}$ is the relaxation time of $j$ th Maxwell spring and a material parameter. For a proof of the thermodynamical consistency of Eqn (10), readers are referred to Hossain [24], Reese and Govindjee [46], Saxena 
et al. [50]. A more advanced nonlinear evolution equation could be taken here. However, the data illustrated in Section 2 do not possess significant nonlinear viscosities as in Amin et al. [3]. Hence, more complex strain energy functions in calculating both equilibrium and non-equilibrium stresses with a linear evolution (Eq. 10) will be sufficient to capture the experimental data. Moreover, our next target is to implement the thermo-viscoelastic model in a finite element (FEM) code, in which a linear evolution law presented in Eq. 10 is relatively straight forward to implement.

\subsection{One-dimensional formulations of the model}

As mentioned in Section 2, all experiments are conducted maintaining a homogeneous mode of deformation. For uniaxial tests, stretches are applied in only one direction. This implies $\lambda_{1}=\lambda$ and rest of the directions are unconstrained. The so-called incompressibility condition at fixed temperature is a widely used assumption for rubber-like materials. Such as a condition results in $\operatorname{det} \boldsymbol{F}=\operatorname{det} \boldsymbol{F}_{v}=\operatorname{det} \boldsymbol{F}_{\Theta}=1$, and it helps to obtain the deformation gradient $\boldsymbol{F}=\left\{\lambda ; \lambda^{-1 / 2} ; \lambda^{-1 / 2}\right\}$. Similarly, the viscous deformation gradient will become $\boldsymbol{F}_{v}=\left\{\lambda_{v} ; \lambda_{v}^{-1 / 2} ; \lambda_{v}^{-1 / 2}\right\}$. With the help of Eq. 7 , the elastic stress is derived as

$$
P_{e}=\left[\frac{\Theta}{\Theta_{0}}+g_{e}(\Theta)\right]\left[2 a+8 b\left[2 \lambda^{-1}+\lambda^{2}\right]^{3}+c\left[1+2 \lambda^{3}\right]^{-\frac{1}{2}}\right]\left[\lambda-\lambda^{-2}\right] .
$$

For a complete calculation of Eqn (11), readers are referred to Hossain [23], Steinmann et al. [52]. It is observed in the experiment section that the temperature field does not significantly change the equilibrium stress response which motivates us to take $g_{e}(\Theta)=1-\frac{\Theta}{\Theta_{0}}$. Now, the viscous stress will be obtained from Eqn (9) as

$$
P_{v}=\sum_{j=1}^{N}\left[\frac{\Theta}{\Theta_{0}}+g_{v}^{j}(\Theta)\right]\left[2 a^{j}+8 b^{j}\left[2\left[\lambda_{e}^{j}\right]^{-1}+\left[\lambda_{e}^{j}\right]^{2}\right]^{3}+c^{j}\left[1+2\left[\lambda_{e}^{j}\right]^{3}\right]^{-\frac{1}{2}}\right]\left[\frac{\left[\lambda_{e}^{j}\right]^{2}}{\lambda}-\frac{1}{\lambda \lambda_{e}^{j}}\right]
$$

where, $\lambda^{j}=\lambda_{e}^{j} \lambda_{v}^{j}$. Once one-dimensional forms of both parts of the total stress are obtained, a scalar form of the equation (10) is required. Inserting the expression of the viscous deformation gradient, the evolution equation from (10) becomes,

$$
\dot{\overline{\lambda_{v}^{2, j}}}=\frac{1}{\tau^{j}}\left[\lambda^{2}-\left[\lambda_{v}^{j}\right]^{2}\right], \quad j=1, \cdots, N .
$$

To obtain an update of $\lambda_{v}^{j}$, Eq. 13 needs to be discretized by an appropriate numerical solver. In the case of an Euler-backward scheme, an updated value of $\lambda_{v}^{j}$ can be calculated as

$$
\lambda_{v}^{j}=\lambda_{v}^{j, n}+\frac{\Delta t}{2 \tau^{j}}\left[\frac{\lambda^{2}}{\lambda_{v}^{j}}-\lambda_{v}^{j}\right]
$$

where, $[\bullet]^{k}=[\bullet]\left(t_{k}\right)$ and $\Delta t=t_{n+1}-t_{n}$. A non-linear iterative solver is required to solve Eqn (14). Once, the current value of $\lambda_{v}^{j}$ is obtained, the actual total Piola stress $\left(P(t)=P_{e}+P_{v}(t)\right)$ can be calculated using Eqn (12). 


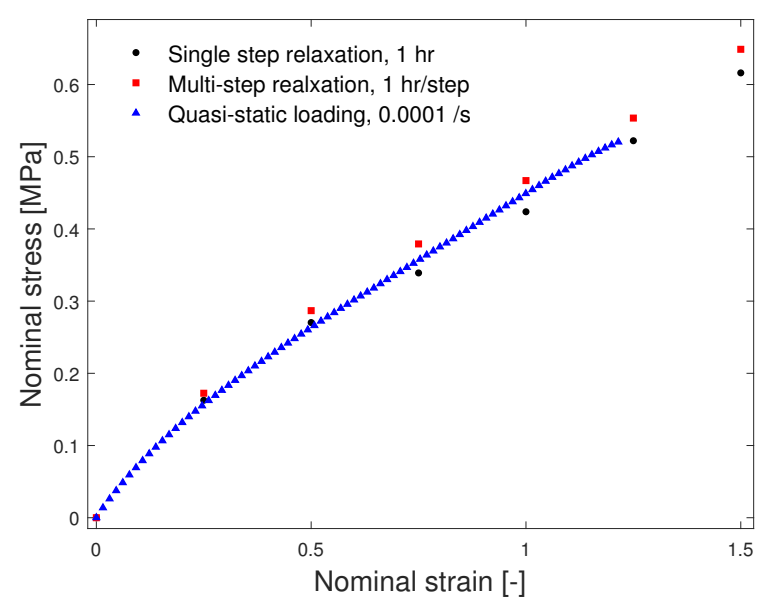

(a)

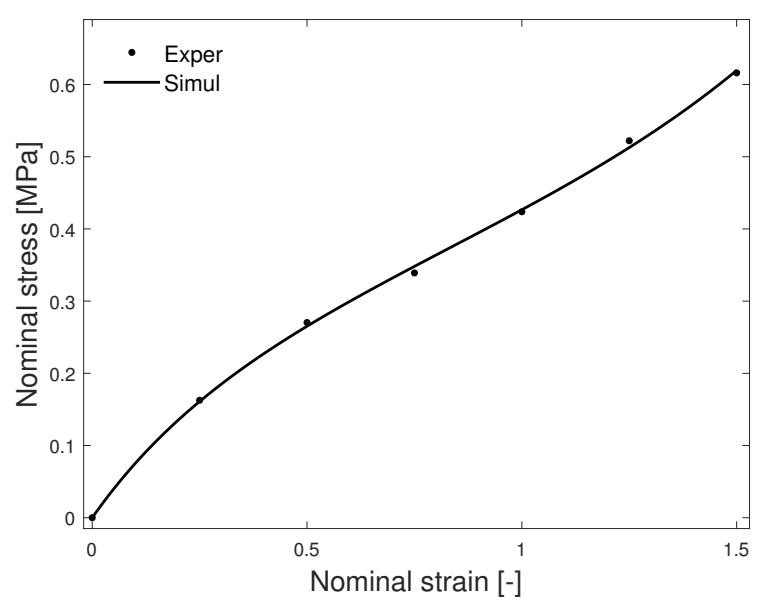

(b)

Figure 16. Elastic parameters identification using equilibrium stress data. (a) A comparison of quasi-static, single- and multi-step relaxation data, (b) Carroll hyperelastic model is calibrated to a set of data originated from single-step relaxation test up to $150 \%$ strain.

\section{Model parameter identification and validation}

In this section, a built-in optimization routine (lsqcurvefit) from MATLAB is used for the parameter identification. With the help of a set of experimental data, elastic, viscous, and temperature-dependent parameters of the thermo-viscoelastic model will be identified subsequently. Afterwards, additional experimental results that are not been used in the parameter identification process will be employed to validate the applicability of the constitutive model. The quality of the fit (also known as the goodness of fit) can be quantified by the calculation of the coefficient of determination $R^{2} \in[0,1]$. It can take a value between 1 , a perfect fit between the experiment and the model, while 0 indicates the worst fit. $R^{2}$, a statistical term which indicates how close the data are to the fitted model, is defined as

$$
R^{2}=1-\frac{\sum_{i=1}^{n}\left[y_{i}-\hat{y}_{i}\right]^{2}}{\sum_{i=1}^{n}\left[y_{i}-\bar{y}\right]^{2}}
$$

In the definition the difference between the values of the experimental results $y_{i}$ and the values of the simulation $\hat{y}_{i}$ are divided by the difference between the values of the experimental results and their average value $\bar{y}$, in which $n$ relates to the number of total data points.

\subsection{Material parameters identification}

\subsubsection{Elastic parameters identification}

Firstly, the identification of the hyperelastic parameters (Eq. 11) is carried out by fitting with time-independent (equilibrium) data. The equilibrium stress data are considered as rate-independent stress responses after the removal of the time-dependent part. In an attempt to remove the time-dependent viscous responses from the stress-strain behaviour of the polymer, three different types of tests are performed in the current study. The equilibrium stresses acquired from the three different experiments are plotted in Fig. 16(a), which show that the three sets of data almost follow a similar trend. Since the relaxed stress data extracted from the single-step relaxation experiments coincide with the quasi-static test findings, these data are adopted for 
Table 1: Elastic parameters of Carroll model identified using equilibrium stress data obtained from the single-relaxation test data

\begin{tabular}{ccc}
\hline $\mathrm{a}[\mathrm{MPa}]$ & $\mathrm{b}[\mathrm{MPa}]$ & $\mathrm{c}[\mathrm{MPa}]$ \\
\hline $2.9312 \mathrm{e}+01$ & $8.6948 \mathrm{e}-01$ & $6.6000 \mathrm{e}-02$ \\
\hline
\end{tabular}

Table 2: Identified viscoelastic parameters for two Maxwell elements with Carroll viscous energy functions

\begin{tabular}{cccccccc}
\hline$a^{1}[\mathrm{MPa}]$ & $b^{1}[\mathrm{MPa}]$ & $c^{1}[\mathrm{MPa}]$ & $\tau^{1}[\mathrm{~s}]$ & $a^{2}[\mathrm{MPa}]$ & $b^{2}[\mathrm{MPa}]$ & $c^{2}[\mathrm{MPa}]$ & $\tau^{2}[\mathrm{~s}]$ \\
\hline $2.253 \mathrm{e}-14$ & $2.243 \mathrm{e}-14$ & $1.016 \mathrm{e}-01$ & $3.209 \mathrm{e}-02$ & $3.374 \mathrm{e}-05$ & $2.224 \mathrm{e}-14$ & $1.193 \mathrm{e}+00$ & $1.632 \mathrm{e}+02$ \\
\hline
\end{tabular}

identifying parameters $a, b, c$. From a family of hyperelastic constitutive models, a three-parameter Carroll model is chosen as it outperforms in predicting hyperelastic data of a wide range of soft polymeric materials, see [21, 52]. Hence, the one dimensional Carroll model from Eq. 11 is fitted with the equilibrium data and the results are demonstrated in Fig. 16(b), where the solid lines are from the model and the circle dots represent experimental data. The Carroll hyperelastic model captures the experimental data accurately. The corresponding identified parameters, $a, b, c$, are shown in Tab. 1. Note that the co-efficient of determination $\left(R^{2}\right)$ of the elastic part is 0.99178 which indicates an excellent correlation of the model with data.

\subsubsection{Viscous parameters identification}

After the identification of the hyperelastic parameters set, viscous parameters appearing in the model (Eq. 12) need to be identified based on a set of time-dependent viscoelastic data. Since the loading-unloading cyclic data of 200\% strain show most pronounced nonlinear stress-strain behaviour (Fig. 7(b)), these data are selected for the viscous parameters identification, namely, $a^{j}, b^{j}, c^{j}, \tau^{j}$. First, it is imperative to identify the number of Maxwell elements that are essential in fitting the nonlinear stress-strain data observed at this strain and strain rate, see Fig. 8. From the computational viewpoints, a reduced number of material parameters is highly desirable. Hence, at first, the data of $200 \%$ strain are fitted with only one Carroll-type Maxwell element and the results are demonstrated in Fig. 17(a). As expected, one Maxwell element (three parameters due to the Carroll energy function) fails to accurately capture the hardening-softening-hardening significantly observed in the stress-strain data. Afterwards, constitutive equations resulted from two and three Carrol-type Maxwell elements (total six and nine viscous parameters, respectively) are fitted with the same data simultaneously taking strain rates of $0.1 / \mathrm{s}, 0.05 / \mathrm{s}$, and $0.01 / \mathrm{s}$. The model calibration with the experimental data are demonstrated in Fig. 17(b) and Fig. 17(c), respectively, where solid lines are simulation curves and the dots are for the experimental data. With two Maxwell elements, the model nicely captures the loading-unloading cyclic data. Note that viscous parameters $c^{1}$ and $c^{2}$ are close to zero, which means the second invariant of the Maxwell elements plays an insignificant role in the model calibration. Overall, there are significant improvements in capturing rate-dependent nonlinearity of the data by two Carroll-type Maxwell elements in comparison to one element. The identified viscous parameters are shown in Tab. 2. Note that the hyperelastic parameters (Tab. 1) are kept frozen during the identification of the viscous parameters. The coefficients of determination $\left(R^{2}\right)$ for the viscous parameters fittings are summarised in Tab. 3 which clearly indicate that the fittings with the two and the three Maxwell elements are better than the one Maxwell element, especially at a low strain rate (e.g., $0.01 / \mathrm{s}$ ). However, there is no significant improvement for the modelling with the three Maxwell elements over two elements. Therefore, we stick to use two Maxwell elements only. 


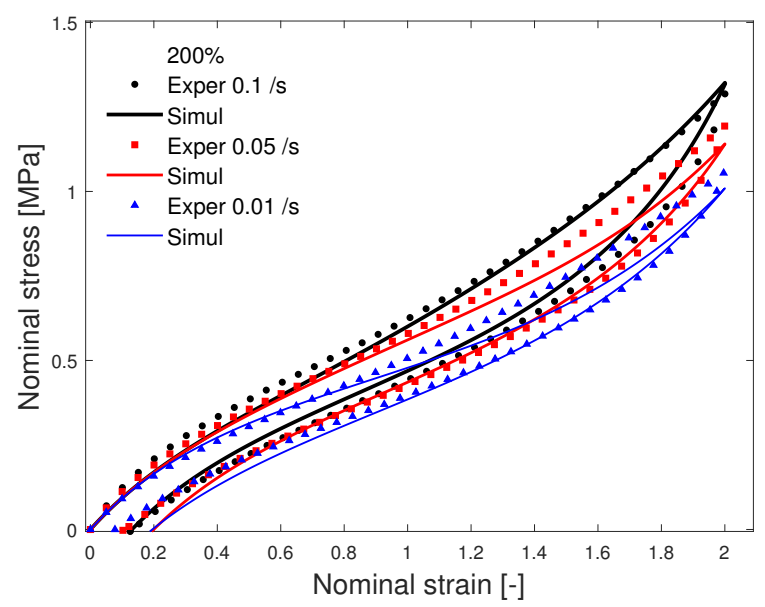

(a)

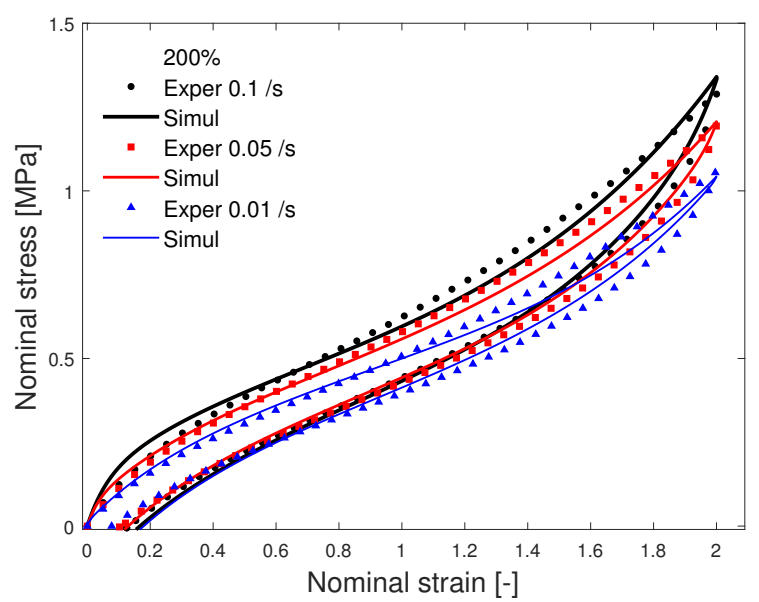

(b)

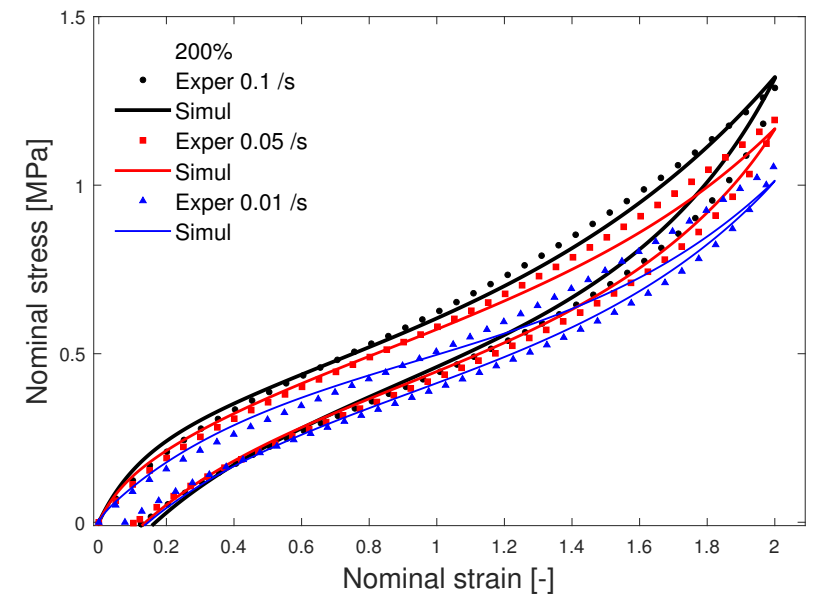

(c)

Figure 17. Viscous parameters identification based on $200 \%$ strain cyclic test data at different strain rates. (a) One Carroll-type element, (b) two Carroll-type Maxwell elements, (c) three Carroll-type Maxwell elements,.

Table 3: Coefficient of determination $\left(R^{2}\right)$ for the fitting at room temperature with varied number of Maxwell elements

\begin{tabular}{cccc}
\hline Strain rates & One Maxwell element (i.e., $N=1)$ & $N=2$ & $N=3$ \\
\hline $0.1 / \mathrm{s}$ & 0.9920 & 0.9920 & 0.9922 \\
\hline $0.05 / \mathrm{s}$ & 0.9909 & 0.9937 & 0.9937 \\
\hline $0.01 / \mathrm{s}$ & 0.9756 & 0.9829 & 0.9829 \\
\hline
\end{tabular}




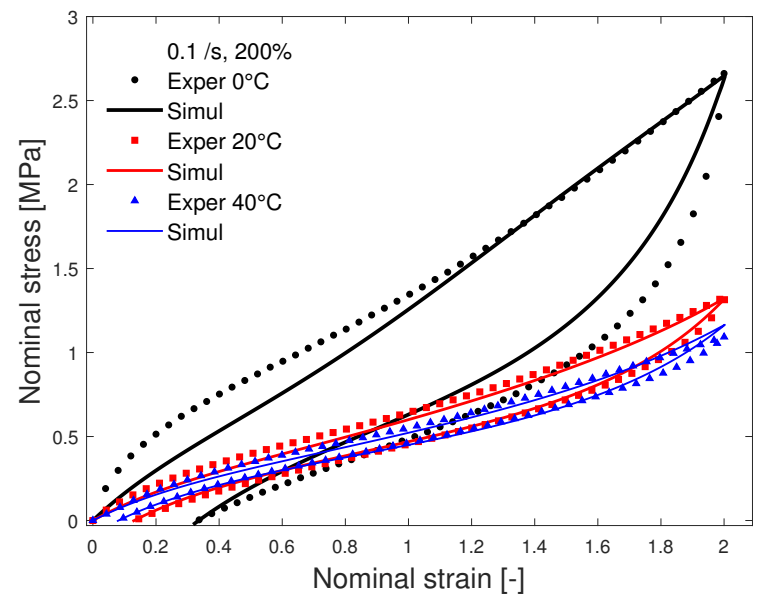

(a)

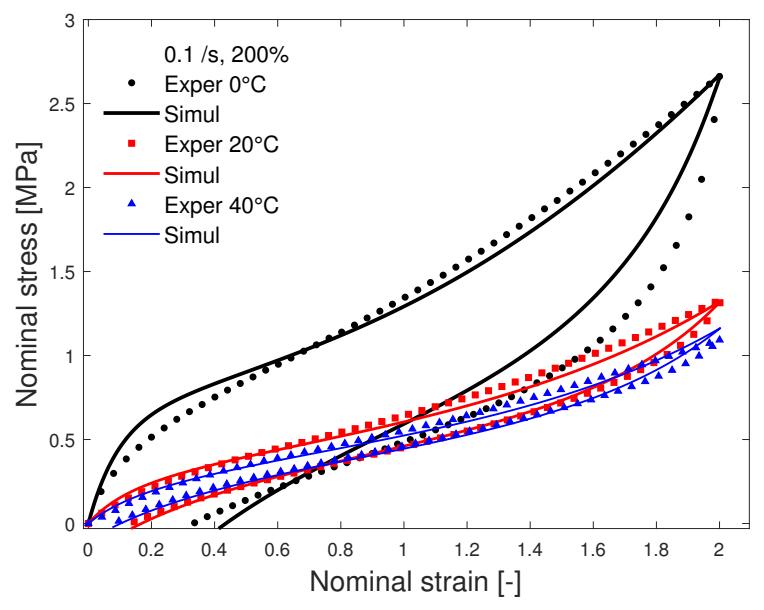

(b)

Figure 18. Temperature-dependent parameters identification based on $200 \%$ strain cyclic test results at 0.1 /s strain rate and different temperature profiles. (a) One Carroll-type Maxwell element, (b) two Carroll-type elements.

Table 4: Temperature-dependent parameters for two Maxwell elements of Carroll-type

\begin{tabular}{ccc}
\hline$a_{T}[-]$ & $b_{T}[-]$ & $c_{T}[-]$ \\
\hline $2.975 \mathrm{e}+01$ & $1.181 \mathrm{e}+00$ & $1.921 \mathrm{e}+00$ \\
\hline
\end{tabular}

\subsubsection{Temperature-dependent parameters identification}

At this stage, both hyperelastic and viscous parameters are identified using the equilibrium stress from the relaxation tests with single steps and the loading-unloading cyclic data of $200 \%$ strain with three different strain rates, respectively. However, all parameters identified and presented in Tables 1 and 2 are obtained by fitting the model with data at the reference temperature, which is room temperature $\left(20^{\circ} \mathrm{C}\right)$ in this study. For identifying temperature-dependent viscous parameters within the modelling framework, the loadingunloading cyclic data for $200 \%$ strain at a strain rate of $0.1 / \mathrm{s}$ are included. Similar to the viscous parameters identification, experimental data of three different temperature profiles, i.e., $0^{\circ} \mathrm{C}, 20^{\circ} \mathrm{C}$, and $40^{\circ} \mathrm{C}$, are simultaneously chosen, see Fig. 9(c). As mentioned in the previous section that temperature is less sensitive in changing the equilibrium stress responses in contrast to its influences on the viscous stresses. Therefore, only viscous scaling functions $\left\{g_{v}^{j}\right\}_{i=1}^{N}$ will be identified using a set of temperature-dependent viscous data and the elastic scaling function $\left(g_{e}+\frac{\Theta}{\Theta_{0}}\right)$ is reduced to unity, i.e., $g_{e}=1-\frac{\Theta}{\Theta_{0}}$. As one Maxwell element fails to capture the viscous data at room temperature, at least two Carroll-type Maxwell elements are required. For these, two scaling functions $g_{v}^{1}$ and $g_{v}^{2}$ need to be determined. In obtaining the best fitting with the data, several exponential functions for $\left\{g_{v}^{j}\right\}_{i=1}^{N}$ are chosen. However, using a trial and error process, we obtain two exponential functions that are best fitted to the experimental data,

$$
g_{v}^{1}(\Theta)=-\frac{\Theta}{\Theta_{0}}+\frac{\exp \left(a_{\mathrm{T}}\left[\frac{\Theta_{0}}{\Theta}-1\right]\right)+b_{\mathrm{T}}}{1+b_{\mathrm{T}}}, \quad g_{v}^{2}(\Theta)=-\frac{\Theta}{\Theta_{0}}+\exp \left(1-\left[\frac{\Theta}{\Theta_{0}}\right]^{C_{\mathrm{T}}}\right) .
$$

After close observations of our previous thermo-viscoelasticity study on VHB [34] and the current study on SIL30, few conclusions can be reached, i) when the non-equilibrium (viscous) stress is hugely influenced by temperature fluctuations compared to the equilibrium stress, and ii) the temperature influence is either 
Table 5: $R^{2}$ values for the model fitting at other temperature profiles

\begin{tabular}{ccc}
\hline Temperature & One Maxwell element $(N=1)$ & $N=2$ \\
\hline $0^{\circ} \mathrm{C}$ & 0.87259 & 0.94937 \\
\hline $20^{\circ} \mathrm{C}$ & 0.97403 & 0.99188 \\
\hline $40^{\circ} \mathrm{C}$ & 0.93670 & 0.99072 \\
\hline
\end{tabular}

Table 6: $R^{2}$ values for the model validation at various strains and strain rates

\begin{tabular}{ccccc}
\hline Strain rates & $200 \%$ & $150 \%$ & $100 \%$ & $50 \%$ \\
\hline $0.1 / \mathrm{s}$ & 0.99226 & 0.99435 & 0.98839 & 0.92040 \\
\hline $0.05 / \mathrm{s}$ & 0.99380 & 0.99034 & 0.99472 & 0.98149 \\
\hline $0.01 / \mathrm{s}$ & 0.98286 & 0.97734 & 0.99196 & 0.99567 \\
\hline
\end{tabular}

exponentially increased or decreased, Equations (16) are particularly suitable. Similar fitting functions for highly temperature-sensitive polymers could be tangent hyperbolic functions as advocated and experimentally fitted by Behnke and Kaliske [5]. Moreover, as depicted in Fig. 11, the temperature-dependent peak stresses show an exponential increase from positive to negative temperature profiles which also gives a motivation to devise exponential-type temperature-dependent functions to capture the influences in the viscous regimes. Within the aforementioned functions, three material parameters, $a_{T}, b_{T}, c_{T}$ are appeared, which are identified by fitting a set of viscous data. The identified parameters are presented in Tab. 4, while Fig. 18(a) presents model fitting with one Carroll-type Maxwell element and Fig. 18(b) illustrates the same with two Maxwell elements. Moreover, $R^{2}$ values for the two and the three Maxwell elements are presented in Tab. 5 which clearly indicate that the model with two Maxwell elements yields improved results over the one Maxwell element. Note that for the reference temperature, room temperature $\left(20^{\circ} \mathrm{C}\right)$ is taken, i.e., $\Theta_{0}=293.15^{\circ} \mathrm{K}$. As expected, one Maxwell element does not have enough flexibility in predicting a wide range of temperature-dependent viscoelastic data. Although one Maxwell element captures loadingunloading loops obtained by performing experiments under different temperature profiles, it fails to capture nonlinearities within the initial strain hardening significantly observed at low temperature profiles and low strains. However, two Maxwell elements capture the exponential increase of stress level with a decrease in temperature. It even captures unloading paths in a relatively good accuracy for all three temperature profiles selected in the identification procedure. Particularly, one Maxwell element largely fails to capture the stressstrain data at $0^{\circ} \mathrm{C}$, while the two elements fairly calibrate nonlinearities. Note that during the identification of $\left\{g_{v}^{i}\right\}_{i=1}^{N}$, the hyperelastic and viscous parameters identified already at the reference (room) temperature and presented in Tab. 1 and Tab. 2, respectively, are kept frozen.

\subsection{Constitutive model validation}

In previous sections, all model parameters are identified by using several sets of experimental data. Now, we will validate the thermo-viscoelastic model. In these cases, loading-unloading cycle data at various strains and strain rates, relaxation data at several strains, and temperature-dependent viscous data will be utilised.

\subsubsection{Model validation: Strain rate-dependent data}

In Sections 4.1.2 and 4.1.3, the viscous and temperature-dependent parameters, respectively, are identified using $200 \%$ strain data for three different strain rates. Now, the model needs to be validated with data 


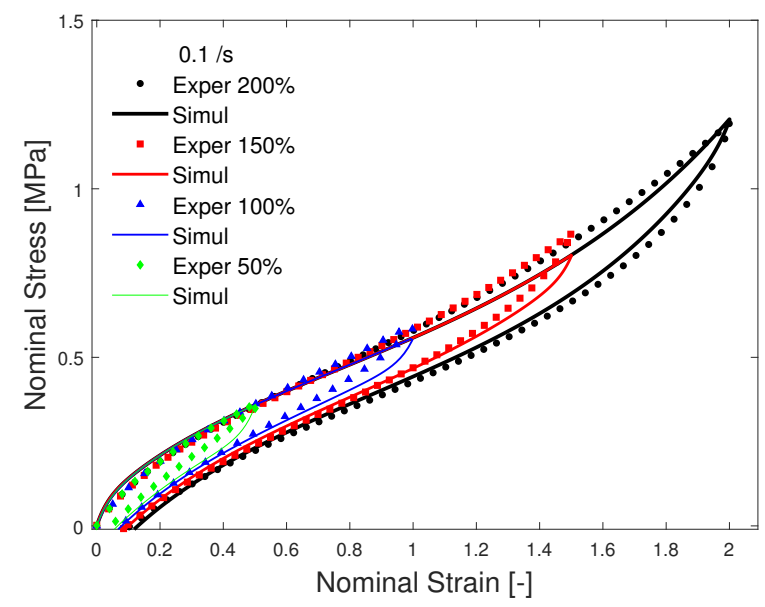

Figure 19. Thermo-viscoelastic model validation with loading-unloading room temperature cyclic data up to $200 \%$ strain at a strain rate of 0.1 /s. The model can perfectly capture the strain-strain data including hardening-softening-hardening observed in SIL30.

Table 7: Coefficients of determination $\left(R^{2}\right)$ for single-step and multi-step relaxations data validations at room temperature

\begin{tabular}{cccc}
\hline Multistep-step relaxation & single-step relaxation (at 125\%) & at 75\% & at 25\% \\
\hline 0.92671 & 0.89409 & 0.96974 & 0.89564 \\
\hline
\end{tabular}

obtained from other strains and strain rates. In this section, all validations are performed with room temperature data, in which loading-unloading cyclic results up to $200 \%$ strains at a strain rate of $0.1 / \mathrm{s}$ are presented in Fig. 19. It is vivid from the figure that the model perfectly predicts a wide range of stress-strain data including hardening-softening-hardening significantly observed in SIL30 polymer. $R^{2}$ values listed in Tab. 6 also substantiate a good prediction of the model with a wide range of experimental data. Furthermore, experimental data of other strain rates such as $0.05 / \mathrm{s}$ and $0.01 / \mathrm{s}$ are also validated the help of the unique set of viscoelastic parameters from Tab. 1 and Tab. 2. Model validations at strain levels of 50\%, 100\%, 150\% are presented in Fig. 20(a), Fig. 20(b), and Fig. 20(c), respectively. Relevant graphs illustrate that, except validation at strain rate of $0.1 / \mathrm{s}$ for $50 \%$ strain, all loading-unloading cyclic experimental data are predicted with a reasonable good accuracy, see also Tab. 6.

\subsubsection{Model validation: Single- and multi-step relaxation data}

Now we will validate single- and multi-step relaxation data with the model. Note that none of the data from the multi-step relaxation experiments was utilised in the parameter identification process. Only equilibrium stresses after relaxed states are partially used in the parameters' identification. From a bunch of relaxation experiments, single-step relaxation data for $25 \%, 75 \%$, and $125 \%$ step strains are used to validate the model, in which Fig. 21(a) presents simulation versus experimental data in a nominal strain-nominal stress format and Fig. 21(b) illustrates the same in a stress versus relaxation time form. Except for a small deviation in following the curved sections of the stress relaxation diagrams at large strains, the model nicely predicts all other experimental data. Furthermore, experimental data from the multi-step relaxation tests are also validated with the model and the comparisons are depicted in Fig. 22. $R^{2}$ values are listed in Tab. 7 which illustrates that the modelling validations with single-step and multi-step relaxation data are less elegant that other validations. However, this can be further improved with strain and strain-rate dependent viscosity functions as recently shown by Hossain et al. [25] for a 3D printed elastomeric polyurethane (EPU). How- 


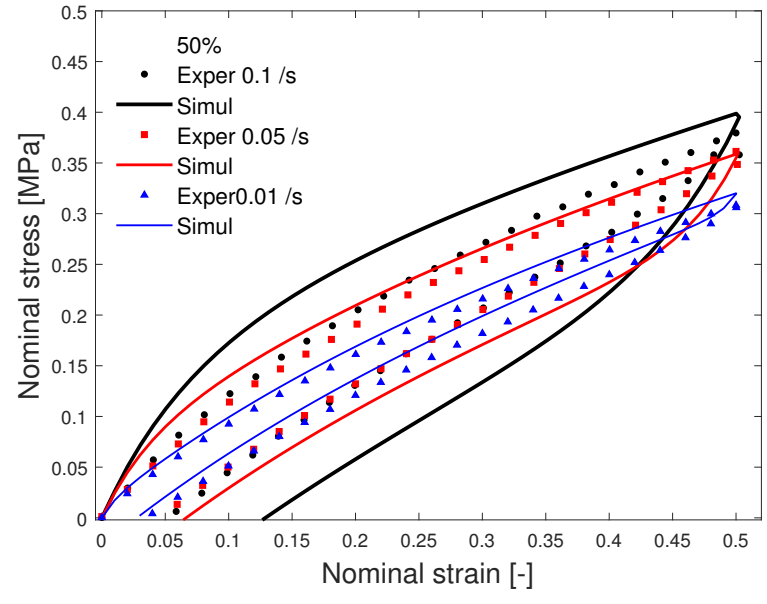

(a)

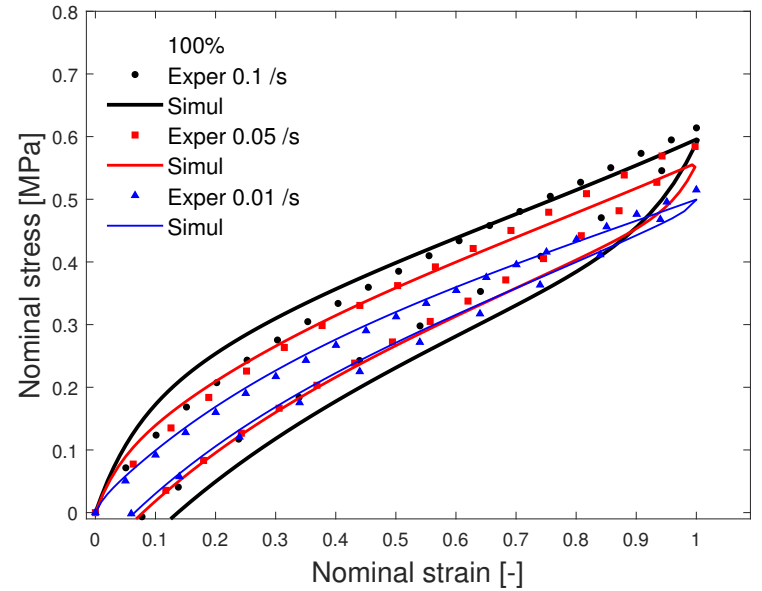

(b)

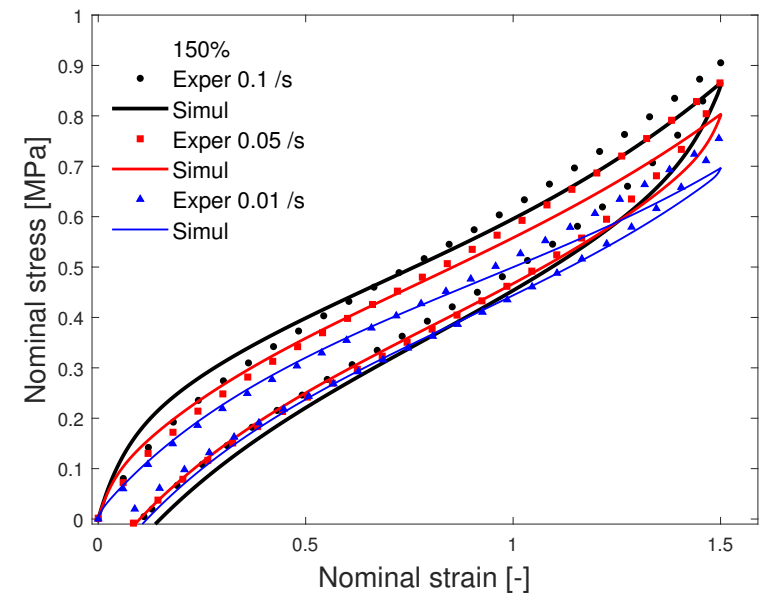

(c)

Figure 20. Model validation by cyclic test data at different strains and strain rates. (a) $50 \%$ strain, (b) $100 \%$ strain, (c) $150 \%$ strain. 


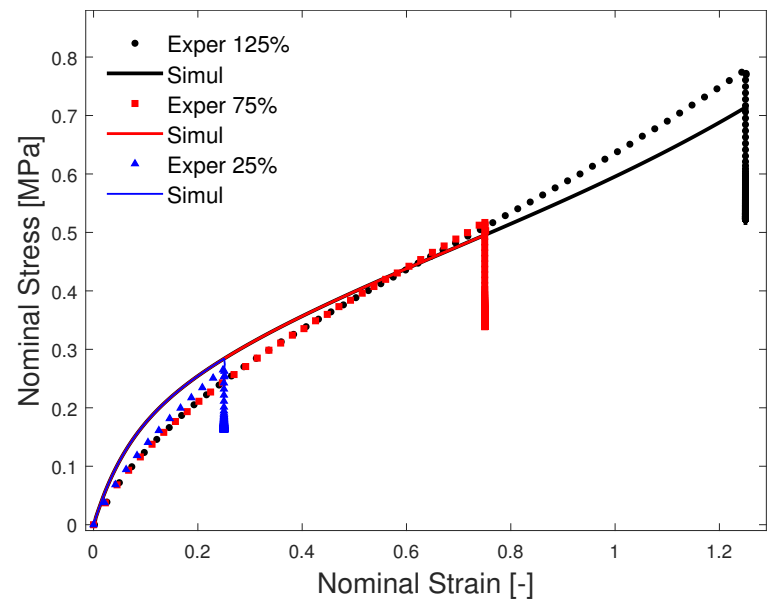

(a)

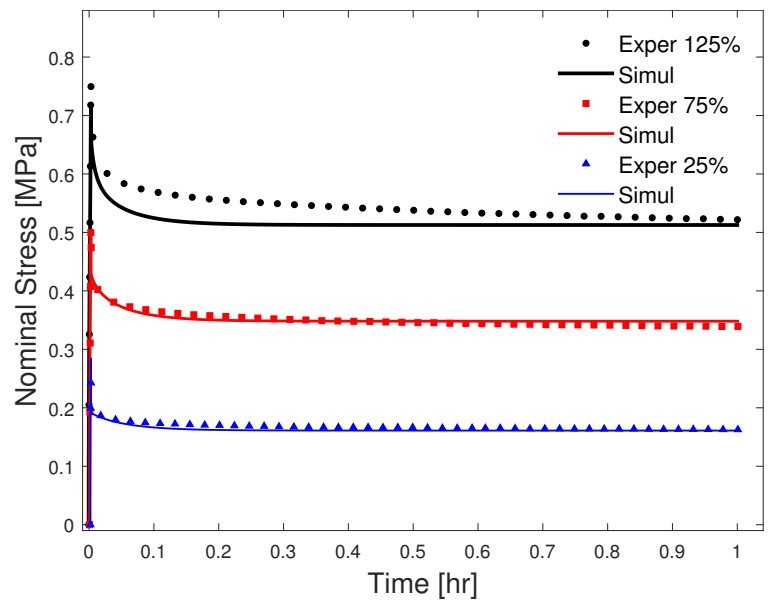

(b)

Figure 21. Model validation by single-step relaxation test data at strains $25 \%, 75 \%$ and $125 \%$. (a) Stress-strain curves, (b) Stress evolution curves.

ever, it is beyond the scope of the current contribution. For the multi-step tests, strain levels beyond $125 \%$ were unsuccessful due to failure in specimens as a result of a long relaxation time. The model predicts the multi-step relaxation data well at strains lower than $100 \%$. However, at higher strain levels, the relaxation time is underestimated, although the equilibrium state is predicted well. Note that all of these validation data are obtained from experiments performed at the reference (room) temperature.

\subsubsection{Model validation : Temperature-dependent data}

Finally, the model is validated with several sets of temperature-dependent data. Fig. 23 and Fig. 24 illustrate model validations with the cyclic data for various temperature profiles ranging from $0^{\circ} \mathrm{C}$ to $60^{\circ} \mathrm{C}$ at strain rates of $0.1 / \mathrm{s}$ and $0.01 / \mathrm{s}$, respectively. Similar to the prediction at room temperature, the stress level is overestimated at the loading path at strain $50 \%$, especially at $0^{\circ} \mathrm{C}$. However, for the temperatures other than $0^{\circ} \mathrm{C}$, the model can predict loading-unloading cyclic behaviour with a good accuracy. In conclusion, we emphasis that with a single set of material parameters (total twelve : three hyperelastic, six viscous, and three temperature-dependent), the model can predict experimental data of a wide spectrum of strains, strain rates, and temperature profiles. The procedure adopted here for the model parameters identification and validation is one of the most widely used techniques. However, at least two more elegant procedures are available in the literature. For instance, Meunier et al. [45] identified model parameters for unfilled silicone polymers using experimental data obtained from homogeneous mode of deformations. For the model validation, they used inhomogeneous boundary value problems in which parameters identified in homogenous modes are used. In this case, the model is required to implement in a finite element framework. Wang and Chester [58] followed a similar approach for parameters identification and model validation in the case of filled polymers. Another costly approach is also available in the literature [31, 40], in which both constitutive parameters identification and model validation were performed using experimental data obtained from inhomogeneous geometries. Since our primary focus of this sequel is to conduct an extensive thermo-mechanical experimental study on a recently invented 3D printed polymer, one of the simplest but most widely used techniques for material parameters identification and the model validation is adopted. Finite element implementation 


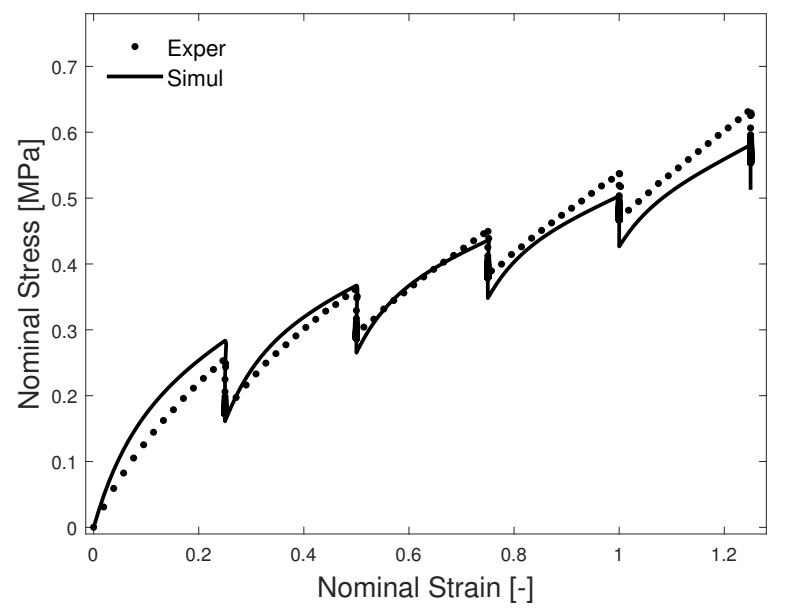

(a)

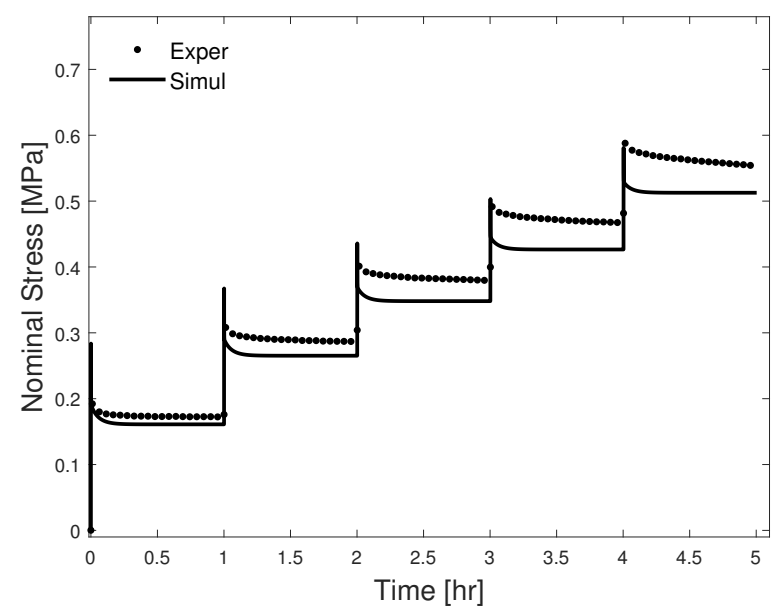

(b)

Figure 22. Model validation by multi-step relaxation test results from strain $25 \%$ to $125 \%$ with a $25 \%$ strain increment between every two steps. (a) Stress-strain curves, (b) Stress evolution curves.

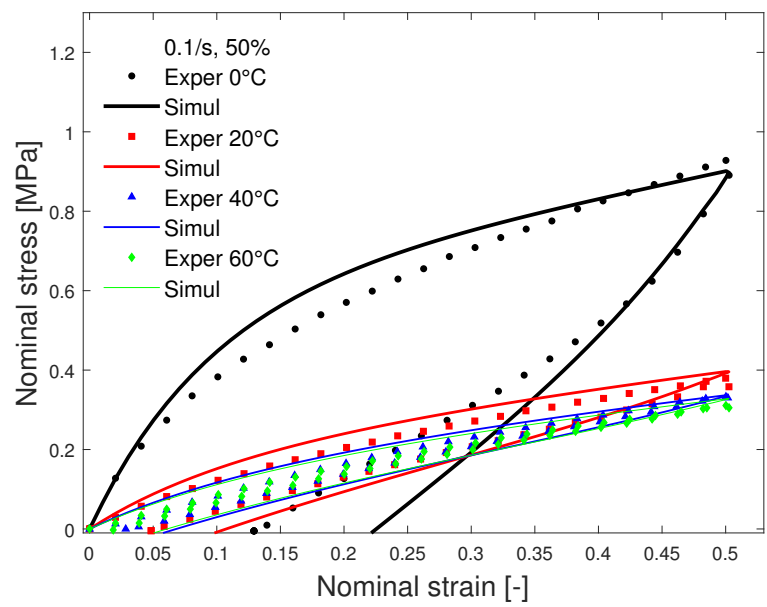

(a)

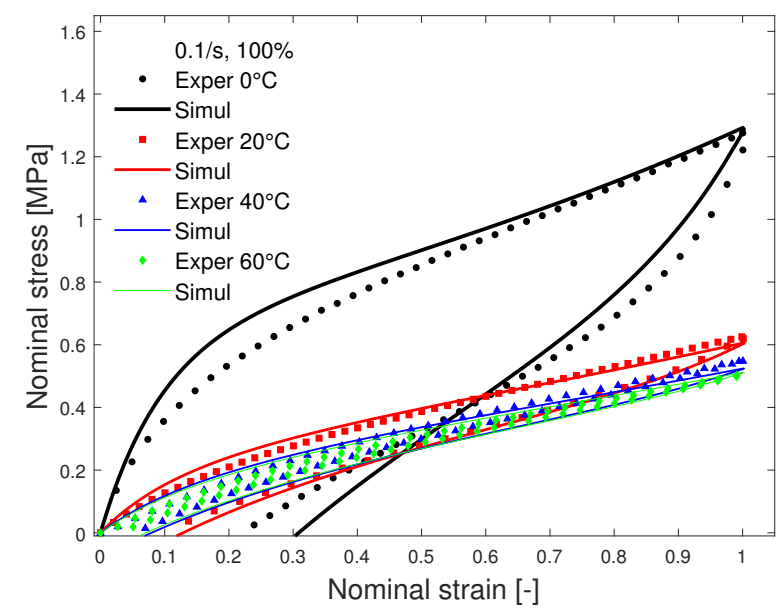

(b)

Figure 23. Temperature-dependence validation by cyclic test data at a strain rate of $0.1 / \mathrm{s}$ with different temperature profiles. (a) $50 \%$ strain, (b) $100 \%$ strain. 


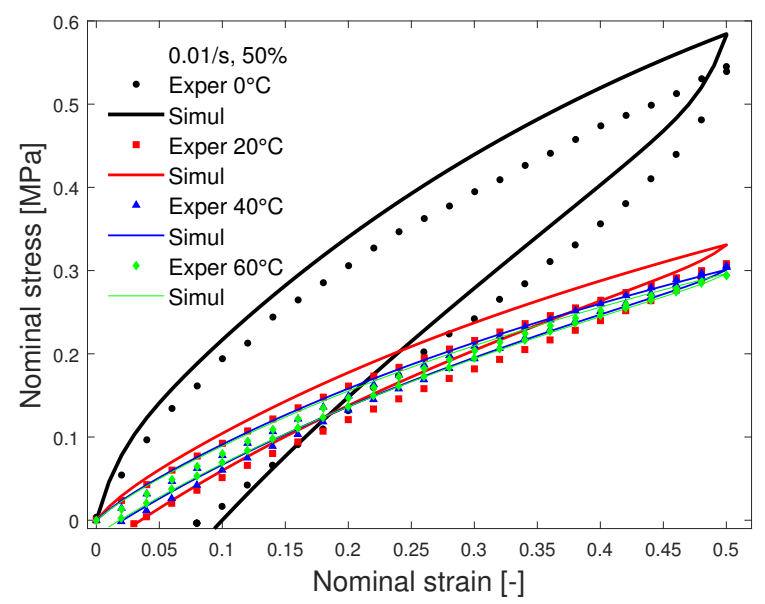

(a)

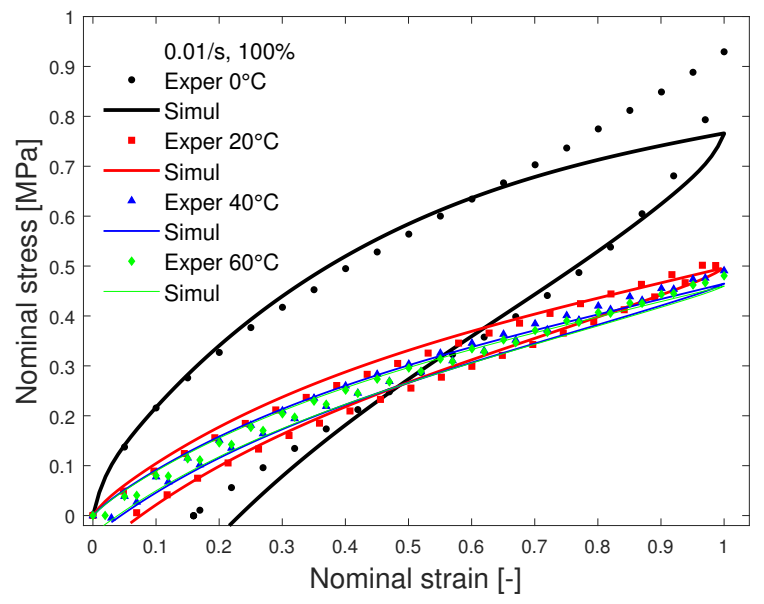

(b)

Figure 24. Temperature-dependence validation by cyclic test results at a strain rate of $0.01 / \mathrm{s}$ with different temperature profiles. (a) $50 \%$ strain, (b) $100 \%$ strain.

of the model and subsequent parameters identification by the aforementioned methods will be addressed in a forthcoming contribution.

\section{Conclusion and outlook for future works}

In this contribution, an additively manufactured elastomeric silicone (SIL30) printed by the UV-curable DLS process is experimentally characterised. All major characterization techniques suitable for a typical soft viscoelastic material are applied. These include Mullins stress-softening tests, quasi-static tests, cyclic tests at different strain rates, single- and multi-step relaxation tests. Relevant experiments demonstrate that the 3D printed silicone SIL30 is a purely viscoelastic polymer with a strong strain rate-dependent behaviour. The UV-curable polymer also shows a relatively large stretchability with rupture occurring at strains above $200 \%$. Furthermore, all major tests are performed under several temperature profiles ranging from $-20^{\circ} \mathrm{C}$ to $60^{\circ} \mathrm{C}$. Most well-known silicone polymers are largely temperature-insensitive. However, the 3D printed SIL30 has high temperature-sensitive responses, in which a higher temperature (above room temperature) softens the polymer significantly and the vice-versa. For instance, single-step relaxation tests at 50\% deformation, the (total) peak stresses are $1.935 \mathrm{MPa}, 0.8263 \mathrm{MPa}, 0.4030 \mathrm{MPa}$, and $0.3326 \mathrm{MPa}$, respectively, for the temperature profiles of $-20^{\circ} \mathrm{C}, 0^{\circ} \mathrm{C}, 20^{\circ} \mathrm{C}$, and $40^{\circ} \mathrm{C}$. For the same deformation, if we compare the normalised equilibrium stresses (actual stresses divided by the respective peak stresses) against the temperature profiles, it can be observed that $82 \%, 60 \%, 33 \%$, and $20 \%$ overstresses disappear, respectively, after one hour of relaxation for the tests performed at $-20^{\circ} \mathrm{C}, 0^{\circ} \mathrm{C}, 20^{\circ} \mathrm{C}$, and $40^{\circ} \mathrm{C}$. This clearly indicates a significant influence of the temperature profile on the material's viscosity. Based on the experimental study presented in the first half of the paper, a thermodynamically consistent constitutive model at finite strains is developed. After parameters' identification, the model is subsequently validated which shows good agreements with data obtained from a wide range of experiments. Additively manufactured SIL30 possesses some unique properties such as biocompatibility, low durometer, and tear-resistance. Hence, 3D printed customised products made of SIL30 become idea candidates in human skin-contact applications, e.g., headphones, wristbands, and various wearable devices. A comprehensive viscoelastic characterization presented 
in this paper will help in selecting appropriate 3D printed silicones. Moreover, the experimentally-driven constitutive modelling of the material can be an essential tool in understanding constitutive responses prior to make a material selection for any particular application. Especially, the digital printing of soft polymers opens new opportunities in creating complex architectured cellular and metamaterials [29], in which the 3D printed silicones such as SIL30 are potential candidates. Note that a Carbon3D manual provides some information regarding SIL30 stretchability and some stress values. However, these are incomplete data whereas the current study reveals more data, particularly its rate-dependences and temperature-sensitivities, which are essential in understanding the material behaviour.

\section{Acknowledgements}

The first author extends his sincere appreciation to the College of Engineering, Swansea University, UK for supporting this work with an Internal Grant Scheme (SURGE).

\section{References:}

\section{References}

[1] R. Adams, S. P. Soe, R. Santiago, M. Robinson, B. Hanna, G. McShane, M. Alves, R. Burek, P. Theobald, A novel pathway for efficient characterisation of additively manufactured thermoplastic elastomers, Materials \& Design, $180: 107917,2019$

[8] B. D. Coleman and M.E. Gurtin, Thermodynamics with internal state variables, Journal of Chemical Physics, 47:597-613, 1967

[9] M. M. Carroll, A strain energy function for vulcanized rubbers, Journal of Elasticity 103: 173-187, 2011 
[10] J. C. Case, E. L. White, R. K. Kramer, Soft material characterization for robotic applications, Soft Robotics, 2(2):80-87, 2015

[11] M. Cianchetti, C. Laschi, A. Menciassi, P. Dario, Biomedical applications of soft robotics, Nature Reviews, 3:143-153, 2018

[12] Resin Data Sheets: CarbonResin SIL30, Carbon3D Company, https: / /www . carbon3d.com/ materials/silicone, 2018

[13] G. Campoli, M. S. Borleffs, S. Amin Yavari, R. Wauthle, H. Weinansa, A. A. Zadpoor, Mechanical properties of open-cell metallic biomaterials manufactured using additive manufacturing, Materials \& Design, 49:957-965, 2013

[14] S. Cheng, B. Chen, Y. Zhou, M. Xu, Z. Suo, Soft sensor for full dentition dynamic bite force, Extreme Mechanics Letters, 34: 100592, 2020

[15] I. Collins, M. Hossain, I. Masters, A review of flexible structures for wave energy converters, European Wave Tidal and Energy Conference Series (EWTEC), 13:1-10, 2019

[16] B. Dippel, M. Johlitz, A. Lion, Thermo-mechanical couplings in elastomers- experiments and modelling, Z. Angew. Math. Mech. (ZAMM), 95 (11):1117-1128, 2015

[17] J. R. C. Dizon, A. H. Espera, Q. Chen, R. C. Advincula, Mechanical characterization of 3D-printed polymers, Additive Manufacturing, 20:44-67, 2018

[18] J. Diani, B. Fayolle, P. Gilormini, A review on the Mullins effect, European Polymer Journal, 45: 601612,2009

[19] C. Duty, C. Ajinjeru, V. Kishore, B. Compton, N. Hmeidat, X. Chen, P. Liu, A. A., Hassen, J. Lindahl, V. Kunc, What makes a material printable? A viscoelastic model for extrusion-based $3 D$ printing of polymers, Journal of Manufacturing Processes, 35: 526-537, 2018

[20] L. Guo, Y. Lv, Z. Deng, Y. Wang, X. Zan, Tension testing of silicone rubber at high strain rates, Polymer Testing 50: 270-275, 2016

[21] M. Hossain, P. Steinmann, More hyperelastic models for rubber-like materials: Consistent tangent operator and comparative study, Journal of the Mechanical Behaviour of Materials, 22(1-2):27-50, 2013

[22] M. Hossain, N. Kabir, A. F. M. S. Amin, Eight-chain and full-network models and their modified versions for rubber hyperelasticity : A comparative study, Journal of the Mechanical Behaviour of Materials, 24(1-2):11-24, 2015

[23] M. Hossain, D. K. Vu, P. Steinmann, Experimental study and numerical modelling of VHB 4910 polymer, Computational Materials Science, 59:65-74, 2012

[24] M. Hossain, Modelling and computation of polymer curing, PhD Dissertation, University of ErlangenNuremberg, Germany, 2010

[25] M. Hossain, R. Navaratne, D. Peric, 3D printed elastomeric polyurethane: Viscoelastic experimental characterisations and computational modelling with nonlinear viscosity functions, In Review, 2020 
[26] R. Hopf, L. Bernardi, J. Menze, M. Zuendel, E. Mazza, A. E. Ehret, Experimental and theoretical analyses of the age-dependent large-strain behavior of Sylgard 184 (10: 1) silicone elastomer, Journal of the Mechanical Behavior of Biomedical Materials, 60: 425-437, 2016

[27] H. Hoeksema, M. De Vos, J. Verbelen, A. Pirayesh, S. Monstrey, Scar management by means of occlusion and hydration: a comparative study of silicones versus a hydrating gel-cream, Burns, 39 (7): 1437-1448, 2013

[28] M. Johlitz, D. Scharding, S. Diebels, J. Retka and A. Lion, Modelling of thermo-viscoelastic material behaviour of polyurethane close to the glass transition temperature, Z. Angew. Math. Mech. (ZAMM), 90 (5):387-398, 2010

[29] J. A. Jackson, M. C. Messner, N. A. Dudukovic, W. L. Smith, L. Bekker, B. Moran, A. M. Golobic, A. J. Pascall, E. B. Duoss, K. J. Loh, C. M. Spadaccini, Field responsive mechanical metamaterials, Science Advances, 5(12):6419, 2018

[30] A. Kumar, K. Patra, M. Hossain, Silicone composites cured under a high electric field : An electromechanical experimental study, Polymer Testing, Accepted, 2020

[31] B. Kleuter, A. Menzel, P. Steinmann, Generalized parameter identification for finite viscoelasticity, Computer Methods in Applied Mechanics and Engineering, 196 (35-36):3315-3334, 2007

[32] Z. Li, Y. Liu, M. Lei, A. Sun, S. Sridhar,Y. Li, X Liu, H. Lu, Y. Q. Fu, B. Xu, A stimuli-responsive gel impregnated surface with switchable lipophilic/oleophobic properties, Soft Matter, 16:1636-1641, 2020

[33] A. Lion, A physically based method to represent the thermo-mechanical behaviour of elastomers, Acta Mechanica 123, 1-25, 1997

[34] Z. Liao, M. Hossain, H. Yao, M. Mehnert, P. Steinmann, On thermo-viscoelastic experimental characterization and numerical modelling of VHB polymer International Journal of Non-Linear Mechanics, 118:103263, 2020

[35] Z. Liao, M. Hossain, H. Yao, Ecoflex polymer of different Shore hardnesses: Experimental investigations and constitutive modelling, Mechanics of Materials, 145: 103366, 2020

[36] Z. Liao, M. Hossain, H. Yao, R. Navaratne, G. Chagnon Mehnert, A comprehensive thermo-viscoelastic experimental investigation of Ecoflex polymer, Polymer Testing, 86: 106478, 2020

[37] L. Liu, Y. Li, A visco-hyperelastic softening model for predicting the strain rate effects of $3 D$-printed soft wavy interfacial layer, Mechanics of Materials, 137: 103128, 2019

[38] S. C. Ligon, R. Liska, J. Stampfl, M, Gurr, R. Muelhaupt, Polymers for 3D printing and customized additive manufacturing, Chemical Reviews, 117:10212-10290, 2017

[39] E. Luis, H. M. Pan, S. L. Sing, A. K. Bastola, G. D. Goh, G. L. Goh, H. K. J. Tan, R. Bajpai, J. Song, W. Y. Yeong, Silicone 3D Printing: Process Optimization, Product Biocompatibility, and Reliability of Silicone Meniscus Implants, 3D printing and Additive Manufacturing, 6(6):319-332, 2019 
[40] R. Mahnken, E. Stein, A unified approach for parameter identification of inelastic material models in the frame of the finite element method, Computer Methods in Applied Mechanics and Engineering, 136(3-4): 225-258, 1996

[41] M. Mehnert, M. Hossain and P. Steinmann, Numerical modeling of thermo-electro-viscoelasticity with field-dependent material parameters, International Journal of Non-Linear Mechanics, 126:13-25, 2018

[42] D. McCoul, S. Rosset, S. Schlatter, H. Shea, Inkjet 3D printing of UV and thermal cure silicone elastomers for dielectric elastomer actuators, Smart Materials and Structures, 26 (12):1-17, 2017

[43] J. Mueller, K. Shea, C. Daraio, Mechanical properties of parts fabricated with injket 3D printing through efficient experimental design, Materials and Design, 86:902-912, 2015

[44] L. Mullins, Softening of rubber by deformation, Rubber Chemistry and Technology, 42(1):339-362, 1969

[45] L. Meunier, G. Chagnon, D. Favier, L. Orgeas, P. Vacher, Mechanical experimental characterisation and numerical modelling of an unfilled silicone rubber, Polymer Testing, 27:765-777, 2008

[46] S. Reese and S. Govindjee, A theory of finite viscoelasticity and numerical aspects, International Journal of Solids and Structures, 35:3455-3482, 1998

[47] T. Rey, G. Chagnon, J. B. Le-Cam, D. Favier, Influence of the temperature on the mechanical behaviour of filled and unfilled silicone rubbers, Polymer Testing, 32 (3): 492-501, 2013

[48] K. K. Reichl, D. J. Inman, Dynamic Mechanical and Thermal Analyses of Objet Connex 3D Printed Materials, Experimental Techniques, 42: 19-25, 2018

[49] M. Robinson, S. P. Soe, R. Johnston, R. Adams. B. Hanna, R. Burek, G. McShane, R. Celeghinie, M. Alves, P. Theobald, Mechanical characterisation of additively manufactured elastomeric structures for variable strain rate applications, Additive Manufacturing, 27 :398-407, 2019

[50] P. Saxena, M. Hossain and P. Steinmann P, A theory of finite deformation magneto-viscoelasticity, International Journal of Solids and Structures, 50 (24):3886-3897, 2013

[51] R. K. Sahu, K. Patra, Rate-dependent mechanical behavior of VHB 4910 elastomer, Mechanics of Advanced Materials and Structures, 23(2): 170-179, 2016

[52] P. Steinmann, M. Hossain and G. Possart, Hyperelastic models for rubber-like materials: Consistent tangent operators and suitability of Treloar's data, Archive of Applied Mechanics, 82(9):1183-1217, 2012

[53] O. A. Shergold, N. A. Fleck, D. Radford, The uniaxial stress versus strain response of pig skin and silicone rubber at low and high strain rates, International Journal of Impact Engineering, 32(9): 13841402,2006

[54] V. Slesarenko, S. Rudykh, Towards mechanical characterization of soft digital materials for multimaterial 3D-printing, International Journal of Engineering Science, 123:62-72, 2018

[55] J. W. Stansbury, M. J.Idacavage 3D printing with polymers: Challenges among expanding options and opportunities, Dental Materials, 32(1): 54-64, 2016 
[56] A. Unkovskiy, S. Spintzyk, J. Brom, F. Huettig, C. Keutel, Direct 3D printing of silicone facial protheses: A preliminary experience in digital workflow, Journal of Prosthetic Dentistry, 120(2):303-308, 2018

[57] A. D.Valino, J. R. C. Dizon, A. H. Espera Jr, Q. Chen, J. Messman, R. C. Advincula, Advances in $3 D$ printing of thermoplastic polymer composites and nanocomposites, Progress in Polymer Science, 98:101162, 2019

[58] S. Wang, S. A. Chester, Experimental characterization and continuum modeling of inelasticity in filled rubber-like materials, International Journal of Solids and Structures, 136-137: 125-136, 2018

[59] M. Wissler, E. Mazza, Mechanical behaviour of an acrylic elastomer used in dielectric elastomer actuators, Sensors and Actuators A 134, 494-504, 2007

[60] T. J. Wallin, J. Pikul, R. F. Shepherd, 3D printing of soft robotic systems, Nature Reviews, 3:84-100, 2018

[61] T. T. Wohlers, T. Caffrey, R. I. Campbell, Wohlers report 2016:3D printing and additive manufacturing state of the industry : annual worldwide progress report, Wohlers Report, 2016

[62] J. R. Tumbleston, D. Shirvanyants, N. Ermoshkin, R. Janusziewicz, A. R. Johnson, D. Kelly, K. Chen, R. Pinschmidt, J. P. Rolland, A. Ermoshkin, E. T. Samulski, J. M. DeSimone, Continuous liquid interface production of $3 D$ objects, Science, 347(6228), 2015

[63] L. R. G. Treloar, The Physics of Rubber Elasticity, Oxford University Press, USA, 1975

[64] M. Yi, B. X Xu, O. Gutfleisch, Computational study on microstructure evolution and magnetic property of laser additively manufactured magnetic materials, Computational Mechanics, 64: 917-935, 2019 